ARTICLE

https://doi.org/10.1038/s41467-019-11063-6

\title{
Lamin A molecular compression and sliding as mechanisms behind nucleoskeleton elasticity
}

\author{
Alex A. Makarov (1) 1, Juan Zou1, Douglas R. Houston', Christos Spanos (1) 1, Alexandra S. Solovyova²,
} Cristina Cardenal-Peralta ${ }^{1}$, Juri Rappsilber (i) ${ }^{1,3} \&$ Eric C. Schirmer (i) ${ }^{1}$

Lamin $A$ is a nuclear intermediate filament protein critical for nuclear architecture and mechanics and mutated in a wide range of human diseases. Yet little is known about the molecular architecture of lamins and mechanisms of their assembly. Here we use SILAC cross-linking mass spectrometry to determine interactions within lamin dimers and between dimers in higher-order polymers. We find evidence for a compression mechanism where coiled coils in the lamin A rod can slide onto each other to contract rod length, likely driven by a wide range of electrostatic interactions with the flexible linkers between coiled coils. Similar interactions occur with unstructured regions flanking the rod domain during oligomeric assembly. Mutations linked to human disease block these interactions, suggesting that this spring-like contraction can explain in part the dynamic mechanical stretch and flexibility properties of the lamin polymer and other intermediate filament networks.

\footnotetext{
${ }^{1}$ Wellcome Centre for Cell Biology, University of Edinburgh, Max Born Crescent, Edinburgh EH9 3BF, UK. ${ }^{2}$ Institute for Cell and Molecular Biosciences/ NUPPA, The Medical School, University of Newcastle, Framlington Place, Newcastle upon Tyne NE2 4HH, UK. ${ }^{3}$ Chair of Bioanalytics, Institute of Biotechnology, Technische Universität Berlin, Berlin 13355, Germany. Correspondence and requests for materials should be addressed to J.R. (email: juri.rappsilber@ed.ac.uk) or to E.C.S. (email: e.schirmer@ed.ac.uk)
} 
C lose to 300 mutations in a single nucleoskeletal protein of largely unknown structure-lamin A - are linked to 13 distinct human syndromes ranging from cardiomyopathy to lipodystrophy and progeria. One hypothesis to explain this wide range of pathologies from mutations in one protein is that mechanical weakening of the nucleoskeleton underlies disease ${ }^{1}$. This hypothesis is supported by observations that nucleoskeletal stiffness affects tissue differentiation, tissue maintenance, and metastatic invasion ${ }^{2-4}$. Yet knowledge of lamin molecular structure and filament assembly-or of other intermediate filaments-is largely limited to electron microscopy ${ }^{5-7}$ and fragment crystal structures ${ }^{8-11}$. For example, for lamin A our insight is limited to crystal structures of the Ig domain representing 1/6th of the protein and two contradictory structures of smaller overlapping fragments at the end of its distended rod domain ${ }^{8-11}$.

Intermediate filaments are the most tensile of the major cytoskeletal systems $^{12}$. Unlike actin and tubulin, intermediate filaments are diverse both within humans who have 70 different intermediate filament genes and in evolution ${ }^{13}$. The main structural feature of intermediate filament proteins is their central distended $\alpha$-helical rod domain, predicted to be made of 3-4 separate coiled coil segments that drive their dimerisation, and is flanked by usually less structured head and tail domains of variable length contributing to further polymerisation $^{14,15}$. Lamins similarly have a short $\mathrm{N}$-terminal region followed by a distended rod domain and a more variable globular C-terminal region. They are likely the progenitor intermediate filament ${ }^{16,17}$ and differ from cytoplasmic intermediate filaments in having an NLS for nuclear targeting, a CaaX box for farnesylation, and additional $\alpha$-helical sequences (6 heptads) inserted into the second coiled coil segment. A recent cryo-electron tomography study of the nucleoskeleton polymer from HeLa cells currently provides our most detailed to date visualisation of the assembled structure of lamins; ${ }^{7}$ however, molecular details of lamin structure, assembly intermediates, and interactions within the polymer remain elusive. In fact, no molecular structure of any full-length intermediate filament protein has been solved due to the flexibility and poor solubility of their predicted central ahelical coiled coil rod domain $8,18,19$.

Here we determine interactions in lamin dimers and tetramers supporting lamin assembly, finding evidence for the predicted parallel coiled coil structure and for intra-molecular compression and inter-molecular sliding supporting nucleoskeletal polymer stress-responses. Specifically, we develop a SILAC cross-linking mass spectrometry (CLMS) approach to distinguish intra- and inter-molecular interactions within lamin homomers: between lamin A molecules within dimers and between lamin dimers at tetrameric assembly stages in solution. This reveals that three flexible linker regions in the lamin A coiled coil rod can electrostatically drive sliding of adjacent coiled coil segments onto each other to achieve a spring-like contraction in rod length. We further show how both head and tail unstructured regions flanking the rod can act as dynamic polar bridges stabilising the lamin A tetrameric interface and likely defining their assembly dynamics ${ }^{20,21}$. Importantly, several tested disease-causing mutations disrupt these properties. Our results suggest an alternative mechanism where unstructured head, tail and linker regions allow reversible small-scale deformation of the nucleoskeletal filaments without the drastic $a$-helix unfolding of the more stable coiled coil segments postulated to govern intermediate filament mechanics 22 . This model explains variations in appearance of nucleoskeletal filaments ${ }^{7}$ and provides a molecular explanation for many uncharacterised disease mutations in lamin A.

\section{Results}

Lamin rod length varies from 40 to $52 \mathrm{~nm}$. A recent cryoelectron tomography study of the intact nucleoskeleton polymer from HeLa cells ${ }^{7}$ suggested a reduction in rod length from the historically measured $\sim 52 \mathrm{~nm}^{5,23}$ to $\sim 40 \mathrm{~nm}$ based on decreased spacing between globular C-terminal densities in the assembled filaments. The difference between in vivo and in vitro measured rod lengths could reflect a change in rod length upon assembly and/or differences in the buffer conditions used for in vitro studies. The mechanism of rod shortening in either case could in theory derive from dimer sliding resulting in increased rod overlap (2-3 nm) $)^{24}$ or from rod shortening by $\sim 15-20 \%$ (Fig. 1a) or from both.

In search of support for the shortening hypothesis we thought to re-analyse distribution of lamin A rod length in conditions favouring lamin dimerisation ${ }^{5,23,25}$ by means of rotary metal shadowing $\mathrm{EM}^{26}$. To tackle observed differences in rod lengths in vitro and in vivo recombinant lamin A dimers purified from bacteria $^{27}$ were equilibrated in either the conventional lamin in vitro dimerization Tris buffer $(25 \mathrm{mM}$ Tris, $250 \mathrm{mM} \mathrm{NaCl}, \mathrm{pH}$ 8.0) or in higher ionic strength sodium phosphate $(\mathrm{NaPi})$ buffer (100 mM NaPi, $250 \mathrm{NaCl}, \mathrm{pH} 8.0$ )-similar to the one used for visualisation of the intact nucleoskeletal polymer ${ }^{7}$. Measuring 300 dimers visualised by rotary metal shadowing EM revealed rod lengths ranging respectively from 40.7 to $56.9 \mathrm{~nm}$ and from 41.0 to 56.0 for $90 \%$ of measurements in Tris and NaPi buffer, with a significant trend towards shorter rods in NaPi buffer: median rod length of $51.1 \mathrm{~nm}$ in Tris buffer vs $49.2 \mathrm{~nm}$ in NaPi buffer ( $p$-value $4.45 \times 10^{-3}$ ) (Fig. 1b, c and Source Data file). This also indicates that buffer conditions can contribute to the rod shortening. A similar shortening was indicated by analytical ultracentrifugation where an increase in more compact species with S-values higher than the $3.81 \mathrm{~S}$ calculated for a dimer with a $51 \mathrm{~nm}$ long rod was observed in NaPi buffer (Fig. 1d). These species are likely also dimeric because, while lamin A head-to-tail tetramers can be clearly observed by EM in either buffer, these are exceedingly rare ( $\sim$ per $20-25$ dimers in NaPi) (Supplementary Fig. 1a and Source Data file); and because a separate population of species with $S$ value roughly fitting the calculated $4.9-5.0 \mathrm{~S}$ for lamin head-to-tail tetramers is readily detectable in analytical ultracentrifugation experiments (Supplementary Fig. 1b). This evidence suggests an existence of compressible elements within the lamin rod.

Cross-linking lamin dimeric and tetrameric assembly states. The observed spread of lamin A rod lengths is a further reflection of its known rod flexibility. Unfortunately there is currently no methodology in place for isolation of dimers in each particular compression state to investigate interactions contributing to these states. Therefore, to better define lamin A structure and investigate whether compression derives from dimer sliding or rod shortening we turned to CLMS. This method is capable of capturing and reporting residue interactions across a population of flexible protein molecules in different structural states as opposed to relying on all protein molecules being in exactly the same state. EDC (1-ethyl-3-(3-dimethylaminopropyl)carbodiimide hydrochloride) was chosen as the cross-linking reagent for this study as one without a linker arm (zero-length cross-linker): it requires respective side chains to be in immediate proximity providing greater cross-linking resolution. It is also heterobifunctional and thus captures actual electrostatic and/or polar interactions within the protein molecule: specifically between side chain carboxyl groups of aspartic and glutamic acid residues and primary amine groups of lysine (and N-terminal methionine) or hydroxyl side chain groups of serine, threonine and tyrosine (residue pairs E/D$\mathrm{K} / \mathrm{S} / \mathrm{Y} / \mathrm{T})$. 

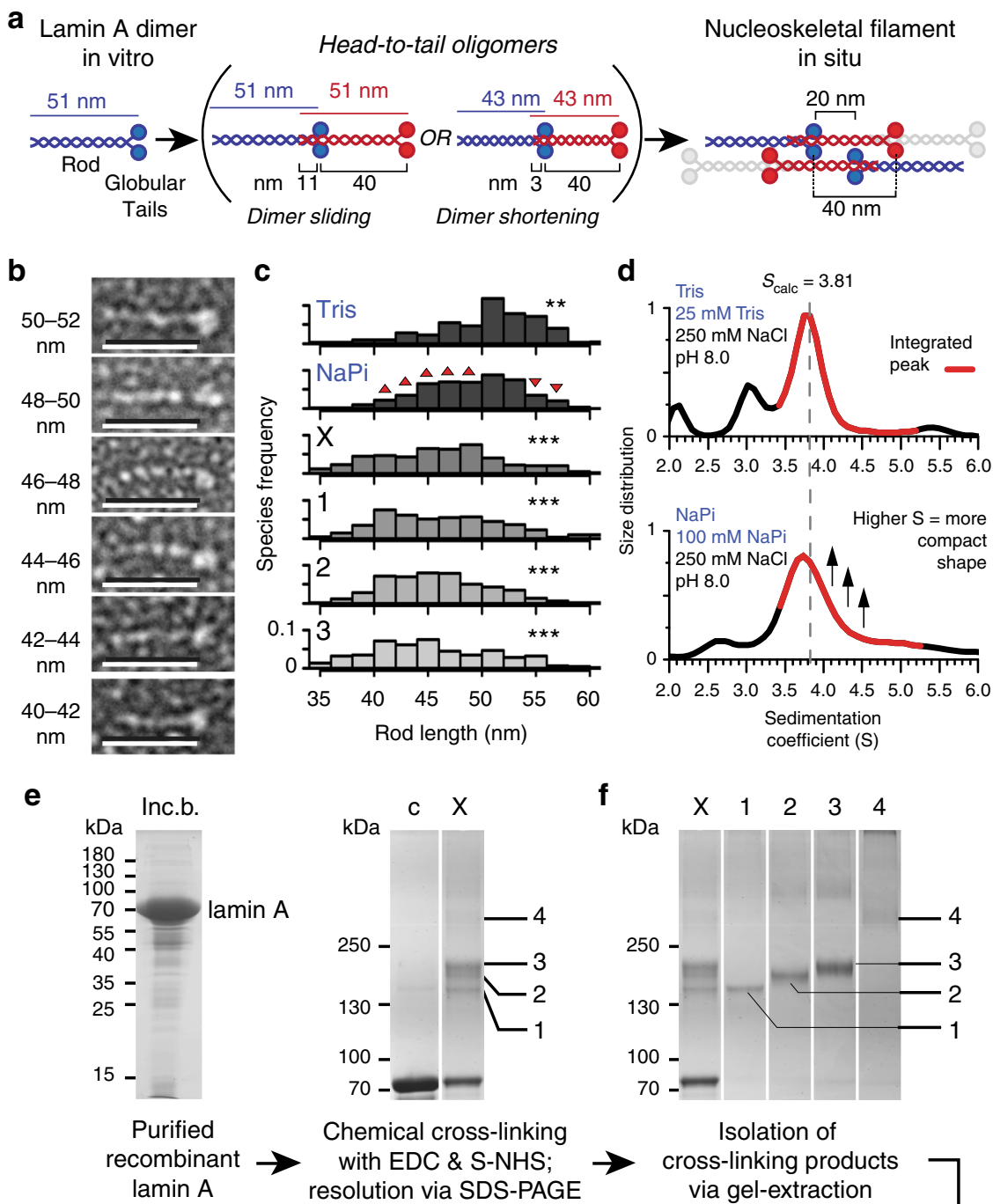
cross-linking products
via gel-extraction

\section{g}
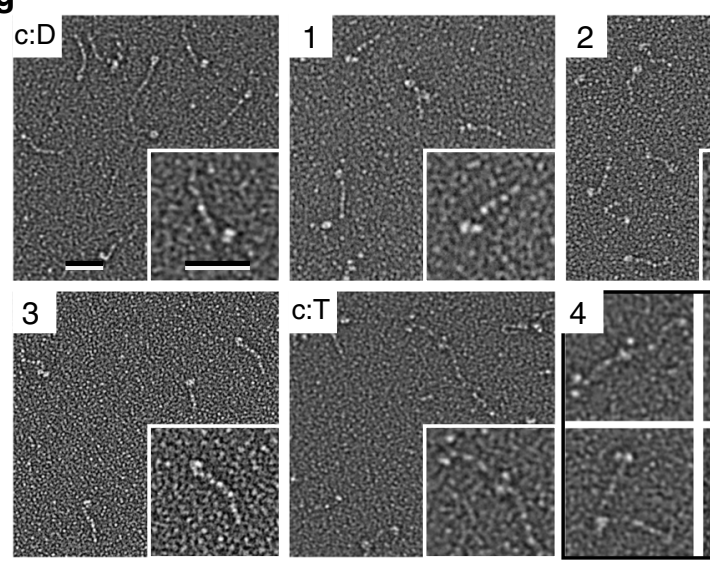

Chemical cross-linking of recombinant lamin A with a mixture of EDC and S-NHS (N-hydroxysulfosuccinimide-a stabilising helper agent) appeared capable of capturing lamin A dimeric structure in its various states of compression as well as its ordered head-to-tail assembly. Cross-linking was carried out in the $\mathrm{NaPi}$ buffer in which we noted the significant variation in the dimer rod length (Fig. 1c). Cross-linking yielded three major productbands (Fig. 1e, bands 1-3) and several minor products (bands 4 and above), which upon isolation by gel-extraction and visualisation with rotary metal shadowing EM (Fig. If, g) were revealed to contain respectively mostly dimeric (1-3) and tetrameric species (4). The latter was expected because head-totail tetrameric species are readily observed alongside dimers in the un-cross-linked control in NaPi buffer (c:T, c:D and Supplementary Fig. 1a). Note that isolated cross-linked species were similar in appearance to those in the un-cross-linked control indicating that cross-linking preserved original lamin architecture. Rod measurements of the dimer-rich cross-linked material further revealed that cross-linked dimers, while widely distributed for lengths, were significantly shorter than those in the 
Fig. 1 Chemical cross-linking captures variation in lamin A rod length. a Rod shortening (middle-left) or increased head-to-tail overlap (middle-right) may explain nucleoskeletal filament characteristics observed in situ. b Example lamin A dimers with rods varying from 40 to $52 \mathrm{~nm}$ visualised by rotary metal shadowing EM. Scale bars, $50 \mathrm{~nm}$. c Frequency distribution of lamin A rod length measured from EM on uncross-linked lamin A in Tris and NaPi buffers, whole cross-linking reaction $(X)$ and individual cross-linked bands in NaPi buffer (1-3) (see $\mathbf{f}$ ). Bars, $2 \mathrm{~nm} ; \mathrm{n}=300$ dimers per condition; $\mathrm{p}$-values (KruskalWallis and Dunn with Holm correction) for differences from NaPi: Tris $4.45 \times 10^{-3}\left(^{\star \star}\right), \times 9.85 \times 10^{-11}\left(^{\star \star \star}\right)$, band-1 $\left.2.43 \times 10^{-7}{ }^{(\star \star}\right)$, band-2 $9.52 \times 10^{-11}$ $\left.{ }^{(\star \star \star}\right)$, band $-32.51 \times 10^{-15}\left(^{\star \star \star}\right)$. d Analytical ultracentrifugation particle size-distributions of lamin A in Tris and NaPi buffers reveals increased frequency of more compact higher S-value species (arrows). e Lamins purified from inclusion bodies (left panel, inc. b.) were chemically cross-linked with a mixture of EDC and S-NHS (middle panel), yielding four major oligomeric products (1-4). f These bands were successfully purified by gel-extraction as evidenced by SDS-PAGE (right panel) of lamin A before (control, c) and after cross-linking with EDC:S-NHS (X) and individual gel-extracted cross-linked bands (1-4). g Rotary metal shadowing EM of cross-linked protein in bands 1-3 reveals principally dimers (control c:D) while band 4 reveals tetramers (control c:T). Source data for $\mathbf{c}$, e and $\mathbf{f}$ are provided in the Source Data file

uncross-linked $\mathrm{NaPi}$ control (Fig. 1c, 1-3), suggesting that interactions leading to rod compression must have been captured by chemical cross-linking and should be identifiable by subsequent mass spectrometry analysis. Similarly cross-linked lamin head-to-tail tetrameric species should, in theory, provide information about the degree of rod overlap and concurrent interactions at the head-to-tail interface.

SILAC chemical cross-linking. To distinguish interactions supporting lamin A homo-dimer subunit assembly and mechanics from those supporting its homo-polymer assembly (i.e. head-totail tetramers), we further developed an original CLMS approach utilising EDC chemical cross-linking and SILAC labelling. Crosslinking of a mix of light and SILAC-labelled heavy lamin A Homo-Iso-Dimers (Fig. 2a, HIDm) enabled distinguishing intrafrom inter-dimeric interactions. Cross-linking of a mix of Homo-/hetero-Iso-Dimers similarly enabled distinguishing intraand inter-molecular interactions within dimers (Fig. 2a, H/ hIDm). Lamin A dimers do not exchange chains even in denaturing conditions of $6 \mathrm{M}$ urea as is indicated by a separate elution of un-tagged and 6xHis-tagged lamin A dimers in Ni-NTA pulldown experiments (Supplementary Fig. 2). Thus to obtain the homo-/hetero-iso-dimer mix we artificially drove chain exchange by refolding gel-extracted monomers, as has been done previously for other intermediate filaments ${ }^{28,29}$. As a proof-of-concept this was again initially attempted on a 1:1 mix of un-tagged and 6xHis-tagged lamin A monomers and formation of hetero-dimers was confirmed in Ni-NTA pull-down experiments showing clear retention of $\sim 50 \%$ of un-tagged lamin A on the beads and its coelution with all of the 6xHis-tagged lamin A (Supplementary Fig. 2a). Co-gel-extraction and refolding of light and heavy lamin A monomers thus results in a homo-/hetero-iso-dimer mix comprised of $50 \%$ of light-heavy and $25 \%$ each of light-light and heavy-heavy dimers (Fig. 2a). An additional in vitro assembly assay was employed to confirm normal structure and assemblycompetence of gel-extracted and refolded lamin A dimers (Fig. 2b).

Chemical cross-linking of both homo-iso-dimer mix and homo-/hetero-iso-dimer mix with EDC/S-NHS yielded identical banding patterns of cross-linked products (Fig. 2c). This allowed a parallel analysis of identical residue interactions in both lamin A samples and consequent identification of their intra-/intermolecular origin: to this end we adapted the comparative crosslinking analysis routine ${ }^{30,31}$ to record the frequency with which cross-links between these residues occurred between pairs of similarly and differently labelled peptides (Fig. 3, "Methods"). In the cross-linking experiment with homo-iso-dimer mix (HIDm $\mathrm{X})$ a residue interaction within a single dimer should always be represented by cross-links between either two light peptides (LL) or two heavy peptides $(\mathrm{HH})$, while an inter-dimeric residue interaction would be represented by cross-links between all 4 combinations of peptides (LL, HL, LH and HH) (Fig. 3b).
Similarly in the homo-/hetero-iso-dimer mix cross-linking experiment (H/hIDm X), a cross-link occurring only between similarly labelled pairs of peptides would be a cross-link happening strictly within a single molecule of lamin A (Fig. 3c) and thus captures an intra-molecular residue interaction. Crosslinks occurring between all possible combinations of peptides would be inter-molecular and occurred between proximal residues either on two opposite chains of a single dimer, or on separate chains of two interacting dimers. Thus, for each crosslink the frequency of occurrence between LL, HL, LH and $\mathrm{HH}$ peptides was recorded as a function of peptide ion intensities in MS1. This was done manually in Xcalibur (Thermo) for all crosslinks or-for a subset of cross-links-semi-automatically using Skyline $^{32}$ across respective extracted ion chromatograms (XiC) (Supplementary Data 1-3) and corresponding frequencies of this particular cross-link occurrence as inter-dimeric and intermolecular were further determined (Fig. 3d). Such analysis of cross-links between the same pair of residues in HIDm X and H/ hIDm X experiments done in parallel thus enables further calculation of the frequency with which these two residues interacted/were proximal as intra-chain or inter-chain within the dimer and between two dimers (Fig. 4 and Supplementary Data 4). A total of 233 unique cross-linked residue pairs (subsequently referred to as just cross-links) from dimer-rich bands 1-3 and 143 cross-links from tetrameric band 4 were identified. Fittingly, SILAC quantification revealed that 229 out of these 233 unique cross-links from dimer-rich bands indeed occurred in dimers with varying frequency while 66 out of 143 cross-links from the tetrameric band were inter-dimeric (Fig. 4).

Structural compression mechanism in the lamin A rod. Intermediate filament rods are thought to consist of multiple $\alpha$-helical segments-coils 1A, 1B and 2-that dimerise into parallel heptad or hendecad coiled coils ${ }^{33-36}$ accounting for $\sim 48 \mathrm{~nm}$ of rod length by prediction. A heptad is a 7 amino-acid motif that drives dimerisation of two alpha helices into a stable twisted superhelix compared to a hendecad which is an 11 amino acid motif that forms a less stable parallel bundle of alpha helices. Most of the lamin A coiled coil rod is predicted to feature heptad structure, but at the end of coil $1 \mathrm{~B}$ and beginning of coil 2 a hendecad structure was predicted to form ${ }^{34,37}$ (Fig. 5a). Individual coils are connected by linkers (L) - both $\alpha$-helical and unstructuredthought to contribute $3.4 \mathrm{~nm}^{38}$, bringing the rod to $\sim 51 \mathrm{~nm}$ (Fig. 5a). A total of 126 cross-links were identified between pairs of residues within the rod and an additional 104-with at least one residue of the pair outside the rod-the head or the tail domains (Fig. 5b, c and Supplementary Data 5, 6). Cross-links within the rod should be informative of its structure, its potential flexible regions and thus of interactions leading to the observed rod shortening. These cross-links were therefore checked against the predicted rod structure starting with those within individual coiled coil segments (see "Methods", Supplementary Discussion). 

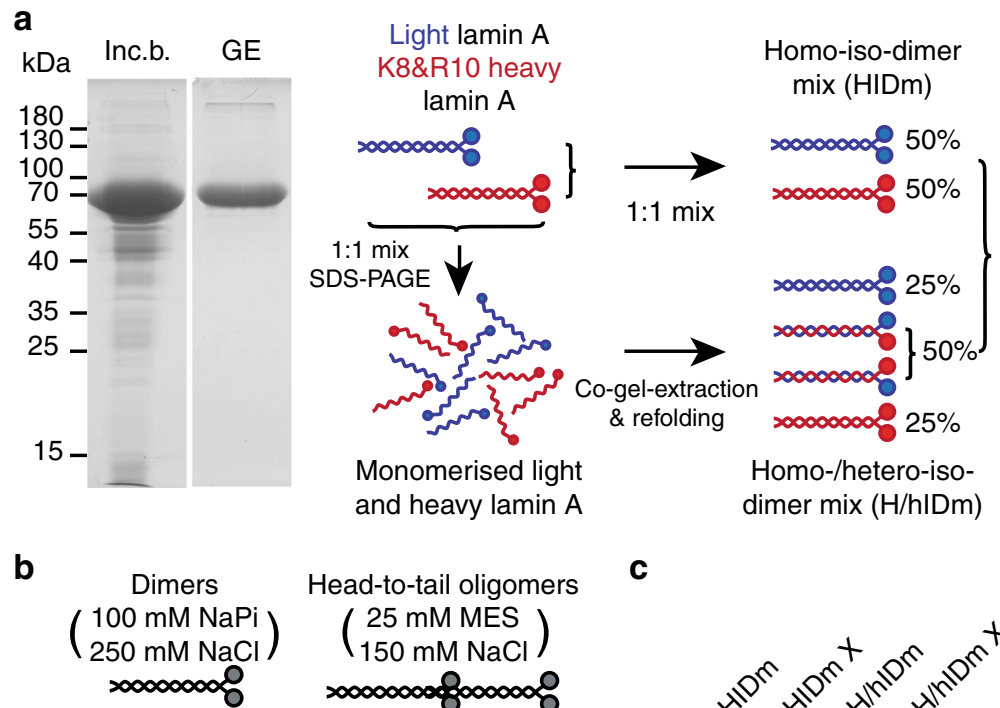

and heavy lamin A
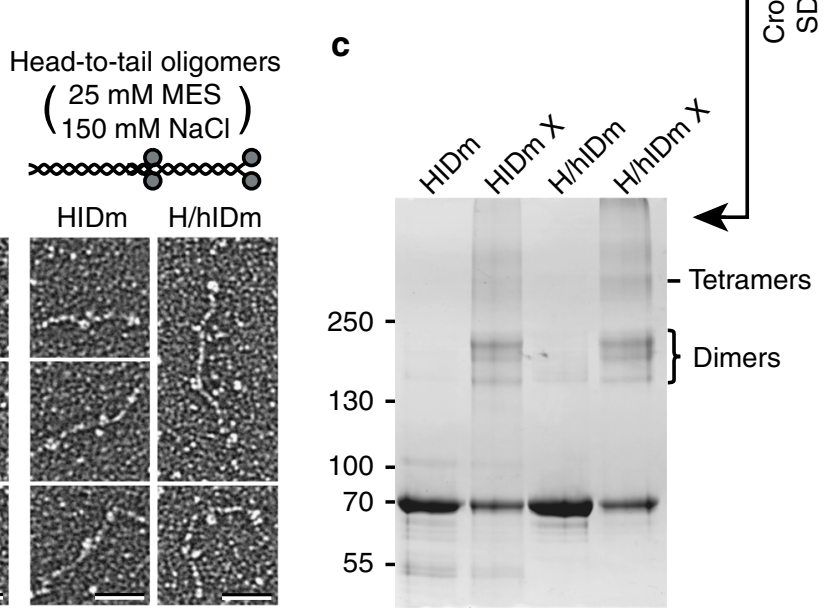

Fig. 2 Lamin A gel-extraction and refolding prior to SILAC CLMS. a Bacterially expressed light (blue) or isotopically-labelled heavy (red) lamins were purified from inclusion bodies (inc. b.). To determine interactions at the inter-dimeric interface a 1:1 light:heavy Homo-Iso-Dimer mix (HIDm) was analysed. To distinguish intra- vs inter-molecular cross-links, the light and heavy lamins were mixed 1:1, gel-extracted (GE), and refolded, generating a Homo-/ hetero-Iso-Dimer mix (H/hIDm) of 1:1:2 light homo-iso-dimers, heavy homo-iso-dimers and light/heavy hetero-iso-dimers mix for cross-linking. b Rotary metal shadowing EM indicates that the gel-extracted and refolded $\mathrm{H} / \mathrm{hIDm}$ lamin A retains structure similar to native HIDm lamin A (micrographs, left column) and both are assembly competent (right row). Scale bars, $50 \mathrm{~nm}$. c Coomassie staining of material before and after cross-linking (X) showing identical cross-linking product patterns for HIDm and H/hIDm lamin A. Source data for a and $\mathbf{c}$ are provided in the Source Data file

A total of 50 such cross-links were identified within the coiled coil segments, 31 of which supported the predicted coiled coils in parallel dimers with some anticipated irregularities in coil segment termini packing: specifically low stability of the coil $1 \mathrm{~A}$ $\mathrm{N}$-terminus ${ }^{39}$ and $\mathrm{PH}$ region ${ }^{40}$, a stutter after $\mathrm{L1}^{38,41}$ and hendecad-heptad transitions in the coil 1B C-terminus and after the $\mathrm{PH}^{35-37}$ (Fig. 6a blue and orange cross-links, Supplementary Fig. 3, Supplementary Data 5 and Supplementary Discussion for irregularities). Importantly, as all of these cross-links either already fit the predicted linear coiled coil structure or can be satisfied via the axial chain rotation and small interruptions in the individual chain $\alpha$-helical structure to bring respective residue side chains in contact for EDC cross-linking, none of these crosslinks can directly account for rod shortening. Interestingly, of the remaining 19 cross-links within individual coiled coil segments, 16 cross-links were between residues in the coiled coil segment termini but too distal for EDC cross-linking in the predicted coiled coil structure even with small structural changes; thus they imply more severe irregularities in these termini (Fig. 6a, red cross-links). Three similar cross-links were also found between residues within in the middle of the coil $1 \mathrm{~B}$. However all of these cross-links could together account for less than half of the observed rod shortening, suggesting that rod shortening cannot be due to just the captured irregularities in coiled coil segment packing.
By contrast and more excitingly, many cross-links were identified between adjacent coiled coils or between linker regions and coiled coils. A total of 76 such cross-links were found at the linker regions L1, L12 and two additional putative linker regions: the once thought classical linker (L2) in the second hendecad of $\mathrm{PH}$ region $^{38}$ and the linker (L3) at the hendecad-heptad transition after the $\mathrm{PH}^{36}$, also previously predicted in ${ }^{42}$ (Fig. 6b, c, Supplementary Discussion). While flexible linker regions could easily fold back onto adjacent coiled coils to satisfy some (though not all) of the linker-to-coil cross-links, most coil-to-coil crosslinks would be too distal for EDC to accommodate in a fully linear rod (Fig. 7a, linker exclusion). Instead these cross-links would require adjacent coiled coil segments to overlap in a relatively parallel tandem stagger or in an anti-parallel fold (Fig. 7a, bottom schematics). Based on the maximal linker extension a group of 55 cross-links tightly clustered in and around the linker regions (Fig. 6b, Supplementary Data 5) were estimated as being possible to satisfy without the rod folding over in an anti-parallel fashion. We thus propose that the observed linear rod shortening may be due to flexible linkers allowing sliding of adjacent coiled coil termini onto one another in a tandem stagger.

To test this possibility, molecular docking in Rosetta with cross-linking constraints (cross-link guided docking) ${ }^{43}$ was employed to search for potential coil termini overlap interfaces 
a

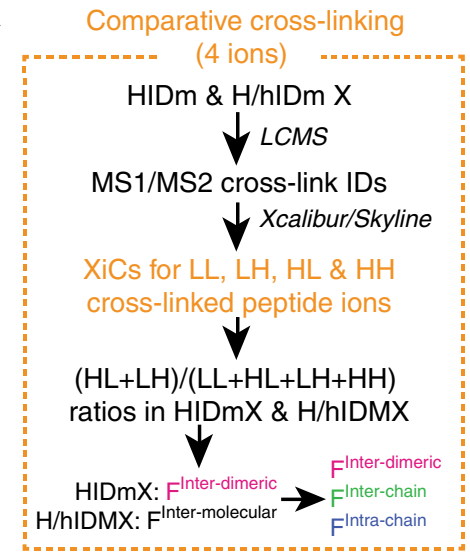

b

HIDm X:

Homo-iso-dimer mix

cross-linking

Intra-dimeric cross-link

1000000008 8000000008

\

LL $x_{x}^{x} x_{x}^{x} x_{x}^{x} \mathrm{HH}$

Inter-dimeric cross-link

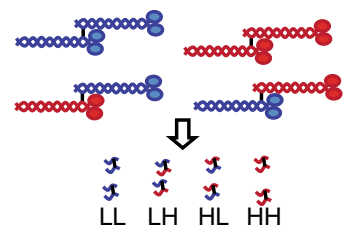

d

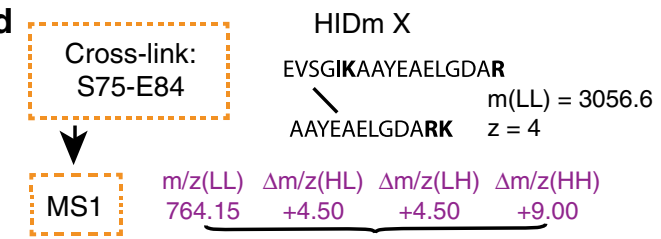

C $\quad \mathrm{H} / \mathrm{hIDm} \mathrm{X}$ :

Homo-/hetero-iso-dimer mix cross-linking

Intra-molecular cross-link

$=$ Intra-chain cross-link

1000000008.00000008

100000008100000008

$\sqrt{2}$

$\operatorname{LL} \int_{x}^{x} x_{x}^{x} x_{x}^{x} H H$

Inter-molecular cross-link
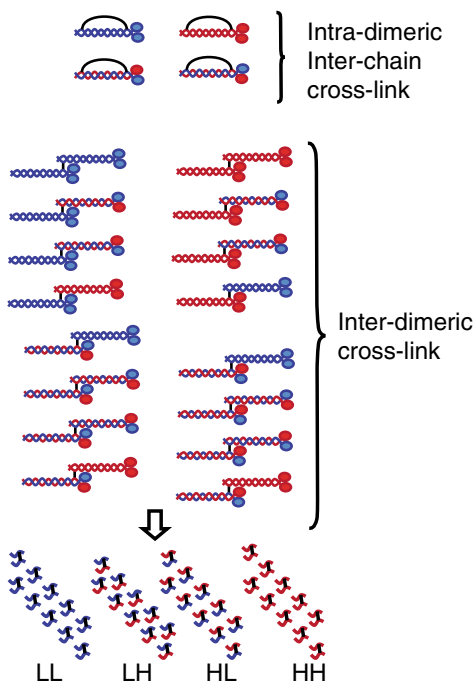

$\mathrm{H} / \mathrm{hIDm} \mathrm{X}$

EVSGIKAAYEAELGDAR

AAYEAELGDAR $\quad m(L L)=2927.46$

$\mathrm{m} / \mathrm{z}(\mathrm{LL}) \quad \Delta \mathrm{m} / \mathrm{z}(\mathrm{HL}) \quad \Delta \mathrm{m} / \mathrm{z}(\mathrm{LH}) \quad \Delta \mathrm{m} / \mathrm{z}(\mathrm{HH})$

$975.82+3.33+6.00+9.33$

4 peptide-pair ion clusters
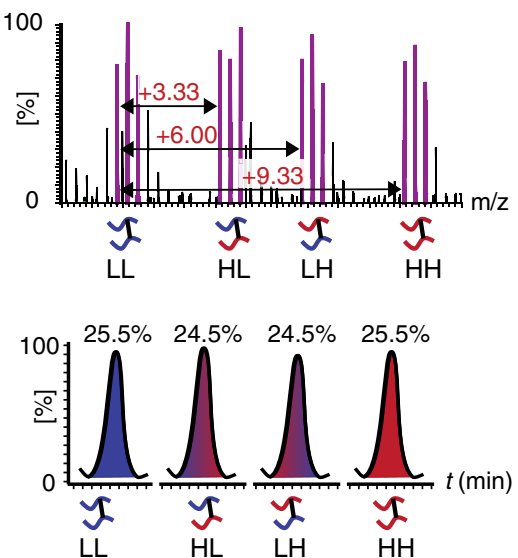

Inter-molecular cross-link

$F^{\text {Inter-molecular }}=2 x \frac{(\mathrm{HL}+\mathrm{LH})}{(\mathrm{LL}+\mathrm{HL}+\mathrm{LH}+\mathrm{HH})}=0.98$ that are relatively stable and would satisfy coil-to-coil cross-links (28 out of 55 ). The main assumption used was that interacting coiled coil termini retain their a-helical geometry while linker regions are flexible. 100,000 structural models featuring docked adjacent dimeric termini fragments for coils $1 \mathrm{~A}$ and $1 \mathrm{~B}$, coil $1 \mathrm{~B}$ and $\mathrm{PH}$, two halves of the $\mathrm{PH}$, and $\mathrm{PH}$ and coil 2 were generated to satisfy sets of cross-links around respective linker regions L1, L12, (L2) and (L3); and Xwalk ${ }^{44}$ was further used to search for models with stable interfaces that satisfied input cross-links (Fig. 7b, see "Methods"). Among these we found both the tandem 
Fig. 3 SILAC CLMS distinguishes inter-dimeric and inter-molecular residue interactions. a The 4-ion comparative cross-linking analysis routine. b, c Massspectrometry of cross-linked and digested HIDm or H/hIDm allows distinguishing pairs of cross-linked peptides/residues originating from respectively intra-/inter-dimeric or intra-/inter-molecular cross-links. Note that inter-dimeric and intra-dimeric-inter-chain cross-links yield similar types of peptide pairs. $\mathbf{d}$ Principle behind the calculation of frequencies of cross-links occurrence between dimers Flnter-dimeric or between molecules Flnter-molecular: for each cross-linked pair of peptides up to 4 precursor ion clusters are found in MS1 of each spectra, each with an $\mathrm{m} / \mathrm{z}$ fitting different degrees of labelling within the cross-linked peptide pair; corresponding extracted ion chromatograms (XiCs) are then determined for each of the mono-, first and second isotopic peak in each cluster-manually in Xcalibur or semi-automatically in Skyline; quantification of peak intensities across XiC areas ions yields information about intra-/inter-dimer/-molecular origin for each cross-link

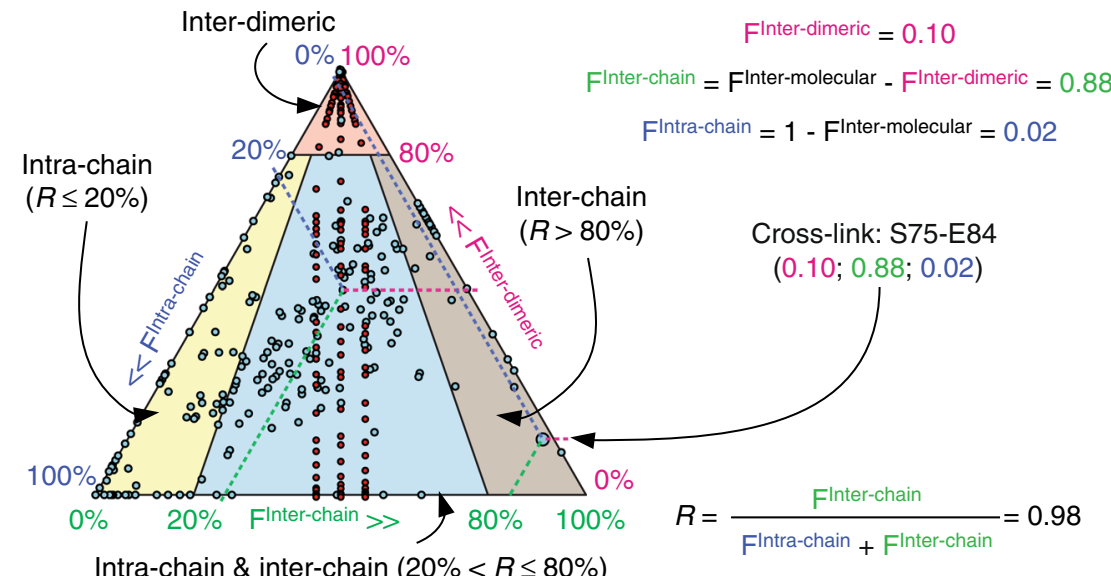

Fig. 4 Mapping lamin A inter-dimeric, inter-chain and intra-chain residue interactions. Frequencies of occurrence as inter-dimeric Flnter-dimeric (magenta axis), intra-dimeric inter-chain Flnter-chain (green axis) or intra-chain FIntra-chain (blue axis) calculated as shown (see also Supplementary Data 4) and plotted on a triangle plot for each cross-link in bands 1-3 and band 4 (see also Supplementary Table 1). Band 4 was analysed only in the HIDm experiment, therefore for only the Flnter-dimeric for these cross-links. Cross-links were deemed as predominantly $(F>80 \%)$ inter-dimeric (red sector), as intradimeric\&intra-chain if $R \leq 20 \%$ (yellow sector), intra-dimeric\&inter-chain $(R>80 \%$, beige sector), intra-dimeric and both intra-\&inter-chain ( $20 \%<R \leq$ $80 \%$, light-blue sector)

stagger models where the linker engages interactions to fold the beginning of the linearly following coiled coil back over the end of the preceding coiled coil and anti-parallel models where the two coiled coils fold back onto one another making roughly a $U$ shape. However, anti-parallel folding models were discounted because these are not consistent with the EM data that shows linear as opposed to folded back rods. Two anti-parallel folds in tandem would maintain the overall rod linearity, but only models with double folds in two of the three-L12, (L2) or (L3)-would not shorten the rod further than any of our measurements. Nonetheless, such possibilities cannot be completely ruled out in the context of an in vitro cross-linking experiment. The wide spread of the measured rod lengths without cross-linking and, more importantly, the downward shift of rod lengths upon crosslinking suggest a temporal nature of any compressed state. Therefore, a population of single-folded intermediate dimers should exist at any given time, which again contradicts collected EM data.

Particular cross-linked residues suggest that both tandem stagger and anti-parallel folding would occur via multiple electrostatic interactions (Supplementary Figs. 4 and 5). The majority of cross-links across L1 and L3 supported tandem stagger rod shortening by up to $5 \mathrm{~nm}$ each with minimal or no rod bending: four out of six coil-to-coil cross-links in both intraand inter-chain variations and 1 only as intra-chain around L1; 11 out of 12 coil-to-coil cross-links around L3 in both intra- and inter-chain variations (see below and the "Methods" section). Some cross-links could obviously not co-exist with others within a single tandem stagger model (Supplementary Fig. 4a), but $>250$ tandem stagger models with stable inter-coiled coil interfaces satisfying overlapping subsets of cross-links were found as determined by Rosetta I_sc values $<-5.0$ (Rosetta's Interface score calculated as a difference between the total energy of the complex and the sum of total energies of each partner in isolation; as detailed in RosettaDock application documentation https:// www.rosettacommons.org/docs) (Supplementary Fig. 4b, 5d and Supplementary Data 7-11). Cross-links also supported rod shortening for L12 with 50\% supporting stable tandem stagger folding ( 3 out of 7 , but 10 out of 20 intra- and inter-chain variations in total) and rod shortening by 3.5 to $4 \mathrm{~nm}$. L2 crosslinks yielded one stable model satisfying only one out of four cross-links and only in the intra-chain variation, arguing against a tandem stagger between the two halves of the $\mathrm{PH}$ region. Together this data clearly shows the possibility of stable coiled coil segment termini tandem stagger interactions that can satisfy observed cross-links and can account for up to $\sim 10 \mathrm{~nm}$ of rod shortening (Fig. 7c).

Such rod shortening is also supported by data from crosslinking-free analytical ultracentrifugation experiments: the integrated peak of sedimentation coefficients (3.4-5.2S) for un-crosslinked lamin A dimers (Fig. 1d) encompasses calculated sedimentation coefficients $\left(S_{\text {cal }}\right)$ for lamin A dimers in various stages of compression calculated using generated tandem stagger models (Supplementary Fig. 6). A multitude of obtained tandem stagger models, especially for adjacent termini of coils $1 \mathrm{~A}$ and $1 \mathrm{~B}$ and for $\mathrm{PH} \mathrm{C}$-terminus and the following part of coil 2, readily point to a high degree of redundancy in any rod shortening mechanism. The true extent of this redundancy became further apparent in an additional in silico docking experiment attempted for adjacent termini of coils 1A and coil 1B using Rosetta in an unconstrained mode: without cross-linking constraints to guide the docking. This generated a much larger set of relatively parallel 
a
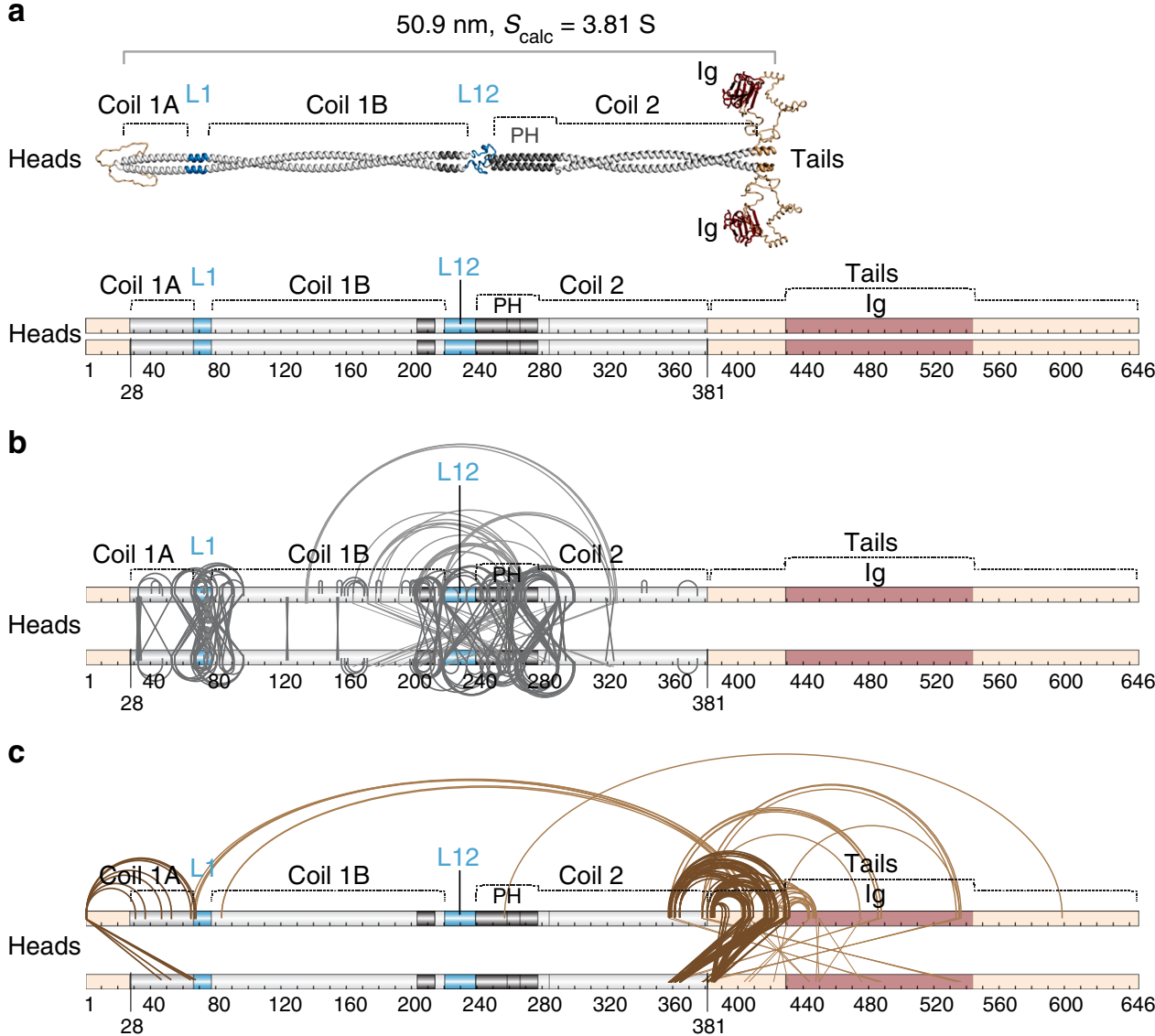

Fig. 5 Distribution of intra-dimeric cross-links between lamin A dimer domains. a Lamin A schematics showing predicted structural organisation (top) and linear domains (bottom). Labels indicate predicted coiled coils (1A, 1B and 2 with PH parallel hendecad region; heptads light grey and hendecads dark grey) and linkers (blue- $\alpha$-helical L1, unstructured L12). b, c Positions of all unique intra-dimeric cross-links identified (Supplementary Table 1) within the rod domain (b) or within the head and tail domains and between these domains and the rod domain (c). Cross-links within the rod appear to cluster around linker regions

stable tandem stagger models, of which almost 800 satisfied one or more of each of the 6 input cross-links in any of their intraand inter-chain variations even when validated with Xwalk set to exclude models requiring additional rotamer manipulation for side-chains of cross-linked peptides (Supplementary Data 12). Similar modelling for dimeric coiled-coil fragments separated by L12 and L3 yielded 506 and 1071 stable parallel tandem stagger models respectively, again satisfying all of intra- and inter-chain variations for all but one of the input cross-links; but only 42 such models for $\mathrm{PH}$ fragments separated by $\mathrm{L} 2$ were found (Supplementary Data 13-15). Together this data provides a potential molecular explanation for observed rod compression/ bending where overlap of individual coiled coil termini is driven by the flexible linkers.

Head and tail domain involvement in lamin compression. Unstructured/flexible regions in the short $\mathrm{N}$-terminal head and globular C-terminal tail domains also flank the rod; so we hypothesised they could similarly fold back over the coil termini to support the head-to-tail inter-dimer interface and sliding. Many intermediate filament head domains are positively charged: the vimentin head folds onto the negatively charged coil $1 \mathrm{~A}^{14,45}$ and is thought to facilitate dimer oligomerisation, presumably via interactions with coil $2^{14,46}$. In lamins both the head and the unstructured part of the tail flanking the rod are positively charged and crucial for head-to-tail oligomerisation ${ }^{21,23}$. Dimer cross-links identified indicate that unstructured regions of the head and tail domains flanking the rod fold onto the adjacent rod termini (Fig. 8a-c). The head N-terminal methionine yielded seven cross-links to multiple coil $1 \mathrm{~A}$ residues that support electrostatic interactions with its primary amine. The rod C-terminus parallels this with two negatively-charged sites in coil 2 yielding 56 cross-links to three positively-charged sites in the flanking unstructured tail region. Paralleling the rod linkers, these charged interactions can support multiple similar conformations to satisfy the observed cross-links (Supplementary Fig. 7 and Supplementary Data 6).

These head and tail interactions with the rod appear to additionally support head-to-tail assembly. Tetramer-band crosslinks connected the head domain $\mathrm{N}$-terminal methionine also to the coil 2 rod C-terminus. Furthermore, the positively-charged sites in the tail that in the dimer cross-linked band were only involved in shortening the rod, in the tetramer band now crosslinked also to negatively-charged coil 1A, L1 and coil 1B regions (Fig. 8d). The switch in interacting residues raises the possibility that the solitary dimer folding primes lamins for a strand exchange that stabilises tetramers (Fig. 8e, Supplementary Fig. 8 and Supplementary Data 6), though some dimer and tetramer cross-links could occur simultaneously (strand coordination). Different cross-links support these models with the two dimers overlapping by 3 heptads $^{24}$ or by all of coil $1 \mathrm{~A}$ (Fig. 8f, g, Supplementary Fig. 9). Charged clusters supporting electrostatic interactions in both models thus could enable rod sliding in the 
a
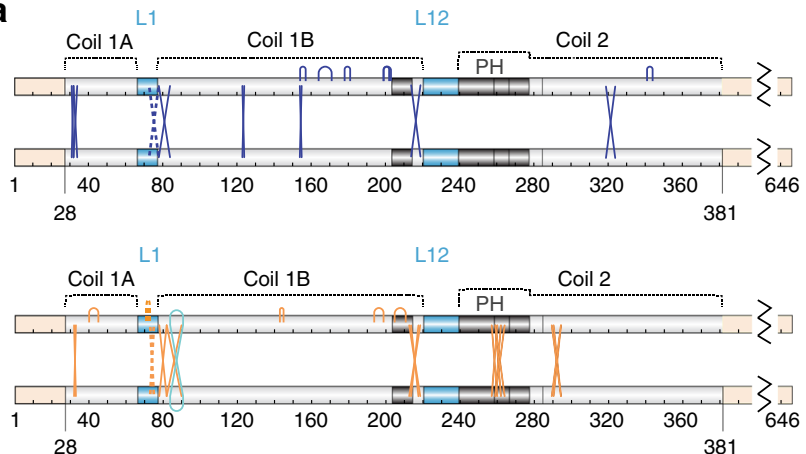

28

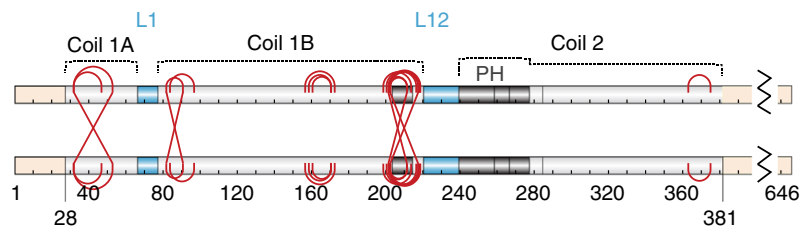

b

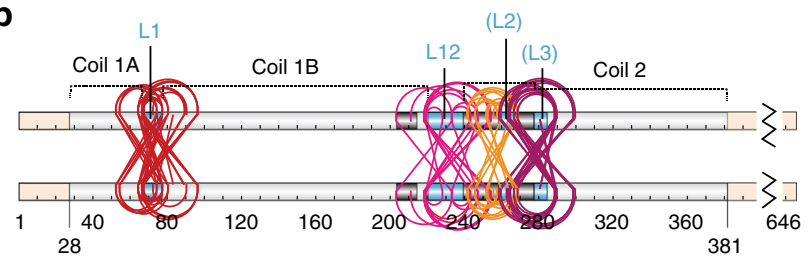

C

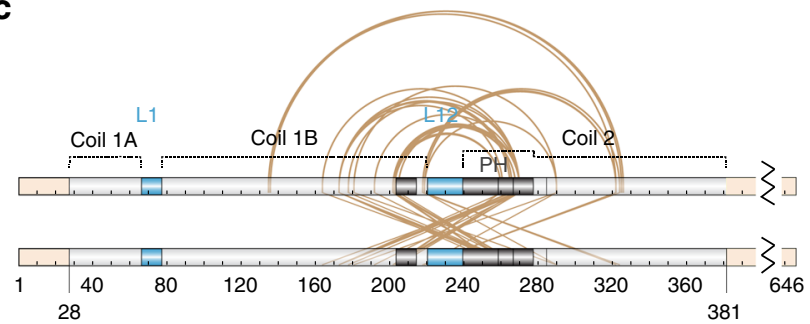

Fig. 6 Categorisation of cross-links in the lamin A rod domain. Cross-links are indicated by the lines: arced lines above indicate intra-chain crosslinks; crossed lines those between-inter-chain cross-links. a Lamin A schematics showing sub-categories of cross-links. A total of 31 cross-links were found in the lamin A dimer rod domain that satisfy classical coiled coil geometry with irregularities in the coiled coil termini and PH packng: 11 intra-chain, 19 inter-chain cross-links (blue and orange lines both) and 1 cross-link occurring both ways (cyan lines). Top: cross-links satisfying the physical constraints of the EDC cross-linker and supporting predicted heptad and parallel hendecad coiled coil structure of the predicted $51 \mathrm{~nm}$ rod. Middle: cross-links that can readily satisfy the cross-linker physical constraints in a parallel dimer with the indicated coiled coil segments, but indicate deviations from the predicted coiled coil structure: axial chain rotation, stutters and hendecad-heptad transition associated irregularities. Bottom: red lines indicate cross-links between residues with side chain at distances exceeding EDC constraints in the predicted structural model. Only such cross-links within the coiled coil regions are shown. b Cross-links around linker regions. Most identified cross-links clustered around L1 (dark red), L12 (light red) and two putative linkers L2 (orange) and L3 (brown) historically annotated within and after $\mathrm{PH}^{36,42}$

(see Supplementary Discussion). c Gold lines indicate all remaining crosslinks within the rod
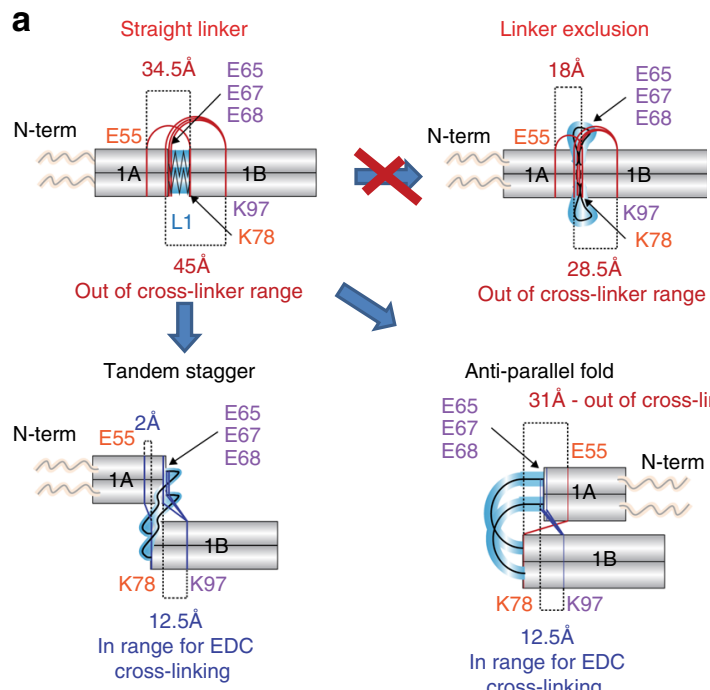

Out of cross-linker range

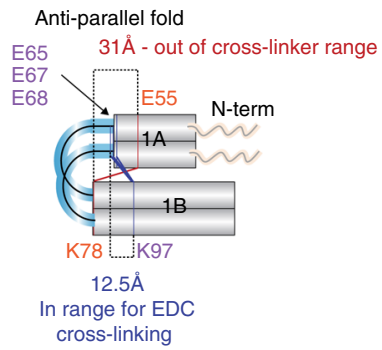

b
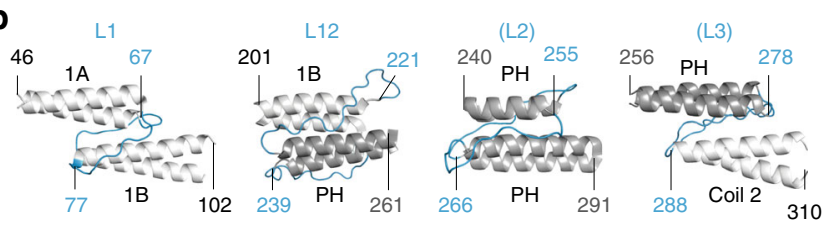

221 models

4 models

1 model

60 models

C

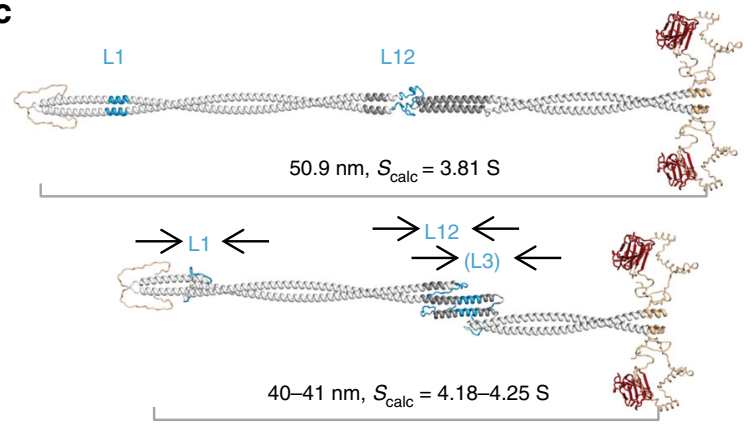

Fig. 7 Modelling of cross-links across linker regions can explain rod shortening. a Principal example models explaining rod shortening around linker L1. Residues in cross-linked pairs E55-K78, E65-K97 (both coil-1A-tocoil-1B) and E67/68-K97 (both L1-to-coil-1B) are too far away from each other (34.5, 48, 45 and $43.5 \AA$ respectively) if $\mathrm{L} 1$ is assumed to be $\alpha$-helical (Straight linker model). Complete exclusion of linker L1 would shorten the rod but is insufficient for bringing cross-linked residues within range for EDC cross-linking (Linker exclusion model). In contrast, tandem stagger folding of the ends of coiled coils over one another or an anti-parallel folding in a U-shape can accommodate the cross-linker constraints and so could explain rod shortening. The tandem stagger model implies a role of the linker in sliding the coiled coil segments onto each other. $\mathbf{b}$ Example tandem stagger models with stable coiled coil termini interfaces (I_sc $<$ -5.0) generated via cross-link-driven docking in Rosetta with Xwalk validation and linkers reconstructed with SWISS-MODEL or MODELLER that support the linear rod shortening via coil sliding. c Model of distended linear lamin A dimer and an example model of dimer compressed via three sequential tandem staggers. When the tandem stagger is applied to three linkers in the rod it can shorten the rod from $50.9 \mathrm{~nm}$ to $40-41 \mathrm{~nm}$, consistent with measurements obtained from analytical ultracentrifugation. $\mathrm{S}_{\text {calc }}$ determined using SoMo (Supplementary Fig. 6 and Source Data file) 
a
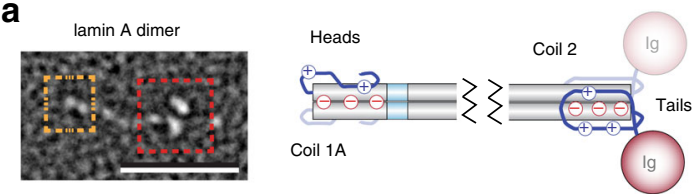

b
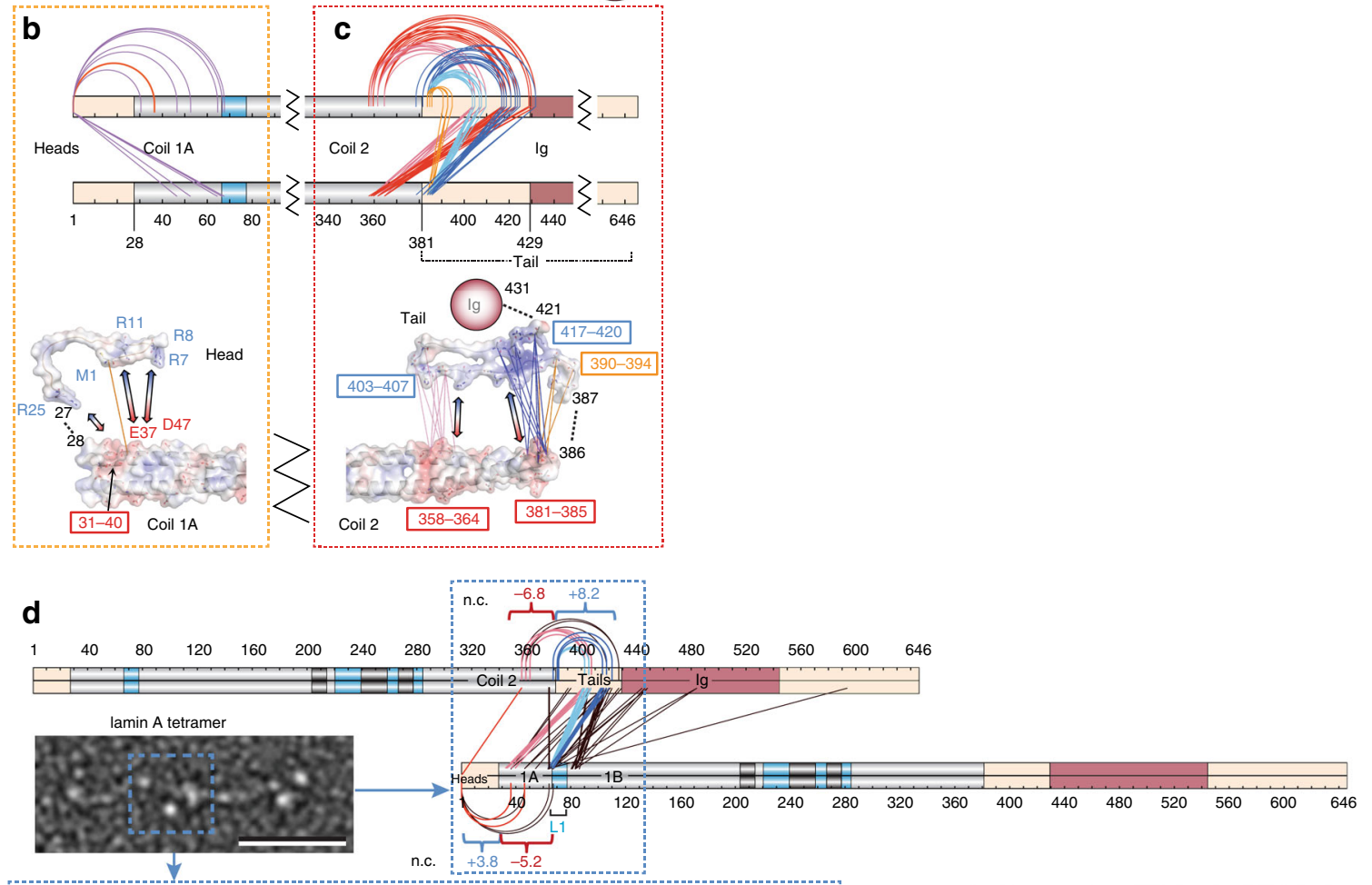

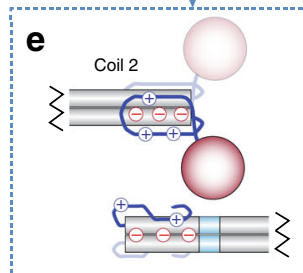

Coil $1 \mathrm{~A}$

f

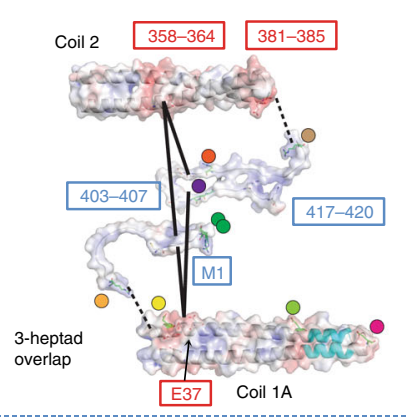

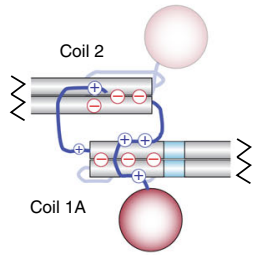

Chain exchange

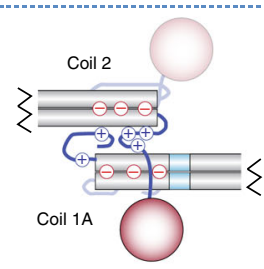

Chain coordination

g

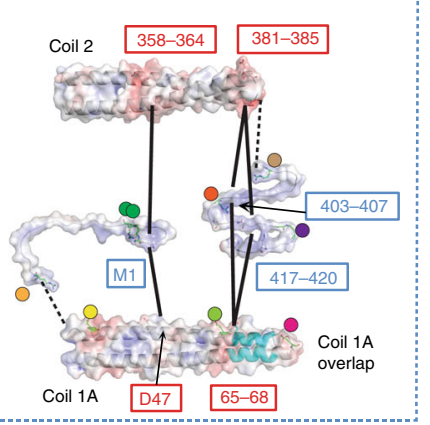

h Inp.

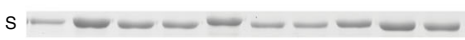

$\mathrm{P}-\mathrm{-}-\mathrm{C}-\mathrm{C}$

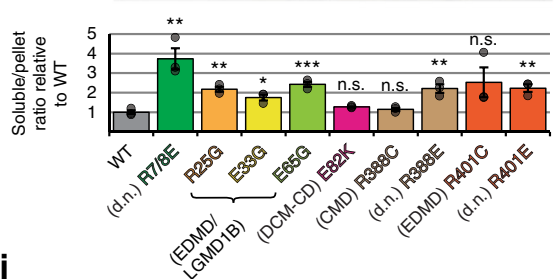

i

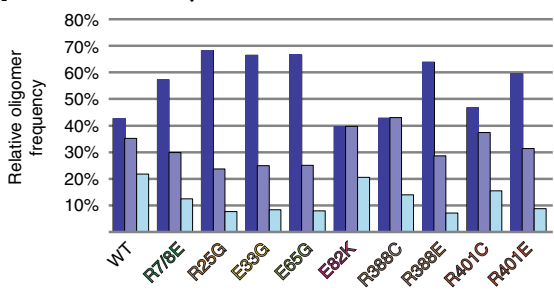

$\square$ Dimers $\square$ Tetramers $\square$ Oligomers

head-to-tail interface as an additional tensile mechanism, consistent with variable distances of C-terminal tail densities in assembled filaments in situ?

Disease mutations disrupt the head-to-tail interface. The proposed electrostatically-driven head-to-tail interface encompasses several residues mutated in patients with Dilated Cardiomyopathy (DCM-CD) or Emery-Dreifuss, Limb-Girdle and Congenital Muscular Dystrophies (EDMD, LGMD1B, CMD). Thus, we tested whether these mutations disrupt the interface using solubility and assembly assays. Substituting rod- flanking region arginines with disease-causing mutations or residues reversing charge (Fig. 8f, g coloured dots) increased solubility in conditions promoting lamin A polymerisation (Fig. 8h). Substituting glutamic acids with glycines in coil 1A achieved similar effects, while such substitutions further away in coil 1B had no effect. Direct examination of assembly stages by rotary metal shadowing EM on $>1000$ particles for each mutant revealed, compared to wild-type, an increase in free lamin A dimers relative to head-to-tail oligomers (Fig. 8i). Thus many disease mutations likely inhibit assembly by disrupting head-to-rod-to-tail interactions. 
Fig. 8 Electrostatic interactions drive head-to-tail docking in lamin A tetramers. a Head and tail domains fold onto their respective rod termini in solitary dimers. b, c This is supported by multiple head-coil 1A (b) and tail-coil 2 cross-links (c). Tail-coil 2 cross-links are coloured according to cross-linked sites (boxed). The head-coil $1 \mathrm{~A}$ cross-link (orange) and two sets of tail-coil 2 cross-links (pink and dark blue) were used to build the example models at the bottom that show head and tail regions, when aligned against adjacent rod termini, can satisfy these sets of cross-links. Electrostatic surface potential reconstructions (red-to-blue colouring- negative-to-positive charges: -5 to $+5 \mathrm{kT}$ ) indicate charged interactions (arrows) drive the folding. $\mathbf{d}$ SILACdetermined inter-dimer crosslinks between the head-coil 1A-L1-1B region and tail-coil 2 region of adjacent dimers in tetramers. Net electrostatic charges ( $\mathrm{n}$. c.) of interacting regions are indicated. e Two proposed modes of lamin A head-to-tail tetramerisation: head and tail strands are exchanged between adjacent dimers termini or interact/coordinate with both. $\mathbf{f}, \mathbf{g}$ Example models of the lamin A tetrameric interface with 3-heptad or full coil 1A overlap. Residues/sites (boxed) with cross-links (black solid lines) and electrostatic surface potentials indicated. Disease-related and de novo (d.n.) mutated residues indicated by dots coloured to match bars and legend colours in panel h. $\mathbf{h}$ In vitro assembly/solubility assay. SDS-PAGE of input (Inp.), soluble (S), and pellet $(P)$ after centrifugation of wild-type and mutant lamins in oligomerisation buffer. Band intensities were quantified for relative soluble:pellet ratios; graph, $n=3$ independent replicas, dots ratios in replicas, bars median replica values, error bars standard errors, $p$-values (T-test) for different from WT:

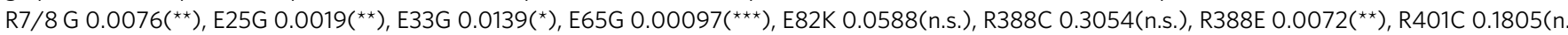
s.), R401E $0.0050\left(^{\star \star}\right)$. Mutations disrupting the interface increase the fraction of soluble material. $\mathbf{i}$ Frequency distribution of free lamin $A$ dimers and dimers incorporated into head-to-tail oligomers determined by rotary metal shadowing EM reveals a similar effect of the mutations blocking the appearance of oligomers; $n=1000$ per mutant. Black dashed lines in all structures schematically connect otherwise directly continuous protein fragments. Source data for panels $\mathbf{h}$ and $\mathbf{i}$ are available in the Source Data file

a

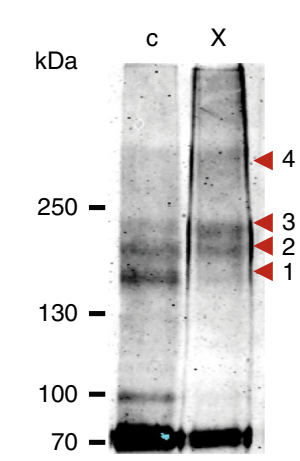

b

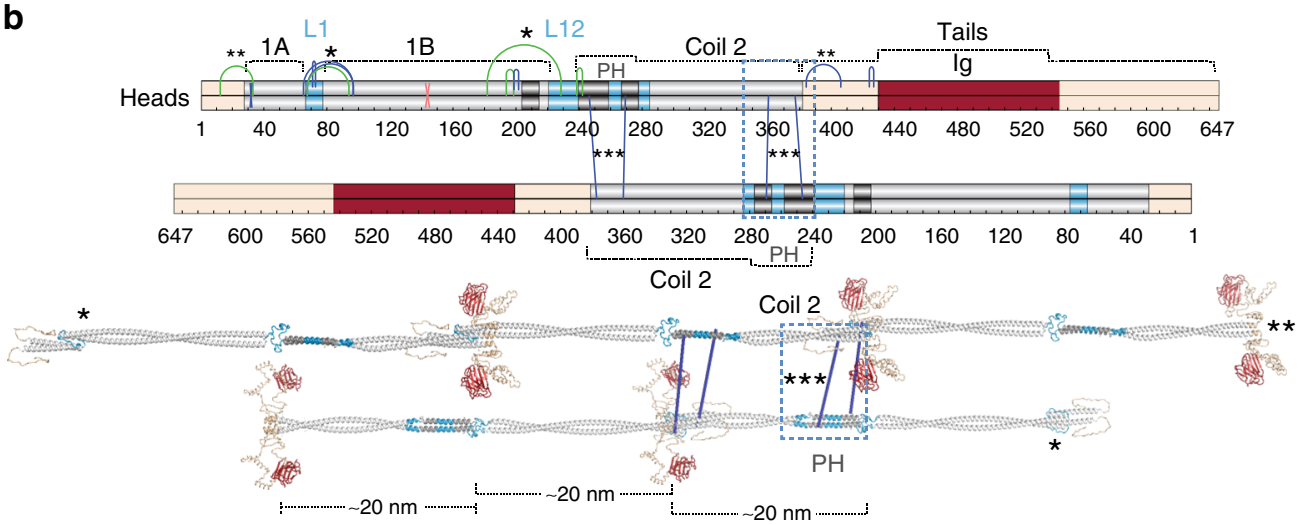

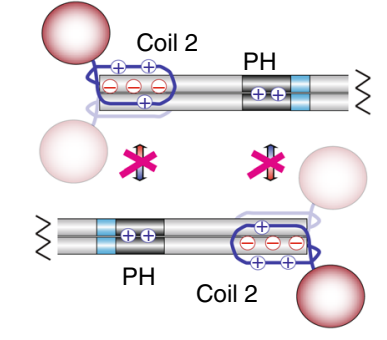

Lateral interaction of dimers blocked e

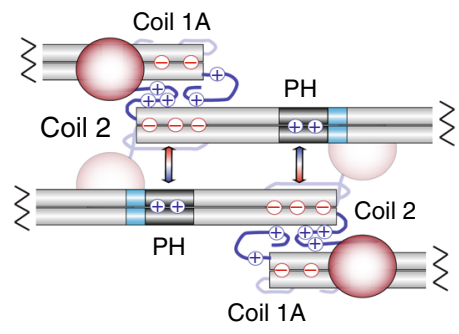

Lateral interaction of oligomers

Fig. 9 Ex vivo nucleoskeleton cross-linking indicates lateral strand assembly. a Lamin A Western blot of uncross-linked and cross-linked rat liver nuclear envelopes. Bands 1-4 appear before cross-linking and changes in band intensities parallel in vitro cross-linking, attesting to successful cross-linking. b Fifteen cross-links (top schematic) identified ex vivo: five were not previously seen in vitro (green) and 10 directly recapitulated in vitro results. Previously in-vitro-encountered cross-links E65-K97 and E68-97 (asterisk) and an additional cross-link E68-S94 all suggest coil 1A and L1 folding over coil 1B, thus recapitulating in vitro conclusions. Similarly, a cross-link D192-S239 indicates L12 and PH folding over coil 1B (asterisk) again recapitulating in vitro findings. Folding of the head and tail onto the adjacent rod termini was recapitulated in cross-link S12-E33 and in the previously seen E385-S407 cross-link (double asterisk). Inter-dimeric K270-E361 and E247-K378 (triple asterisk) cross-links were consistent with a lateral anti-parallel half-staggered dimer interface in filaments (bottom model). One cross-link S143-E145 (red) was strictly intra-molecular in vitro, but was inter-molecular (between overlapping peptides) ex vivo. c Alignment of coil 2 fragments with electrostatic potential overlay: negative (red), positive (blue). Gradient-filled arrows indicate potential weak electrostatic attractions. d, e Differences in spectra recovered between dimeric (d) and tetrameric (e) species suggest that these electrostatic interactions are likely blocked by the tail unstructure regions between dimers, but possible between head-to-tail oligomers. Source data for panel $\mathbf{a}$ are available in the Source Data file

Lamin compression in the intact polymer. We also tested whether these in vitro measured interactions also occur in the intact nucleoskeleton by investigating ex vivo cross-links in rat liver nuclear envelopes. As a general support, the ex vivo crosslinked material yielded a lamin A banding pattern similar to in vitro (Fig. 9a and Supplementary Table 1). CLMS analysis of ex vivo cross-linked material yielded a total of 15 cross-links, 10 of which were also identified in vitro, and collected data further recapitulated the described key in vitro findings. The proposed mechanism for rod shortening via rod/linker tandem stagger folding in L1 and L12 is supported by linker-to-coil cross-links E68-S94, E68-K97 (L1-to-coil 1B) and D192-S239 (L12-to-coil 

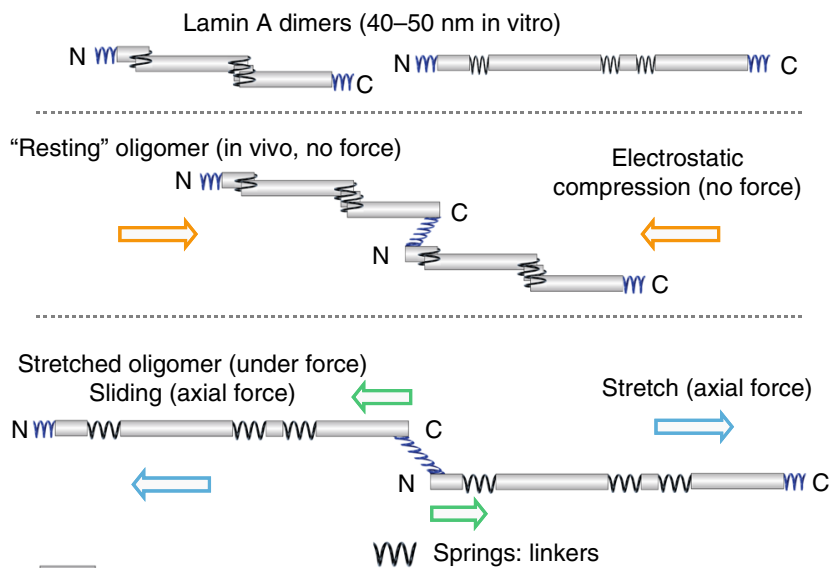

Blocks: coiled-coils $\quad \mathrm{m} /$ Springs: head \& tail regions

Fig. 10 Model for lamin A compression spring. The lamin A dimer rod can compress to $\sim 40 \mathrm{~nm}$ by the tandem staggering of coiled coils or be stable in a semi-relaxed state without the stagger at $\sim 50 \mathrm{~nm}$ (uppermost schematics). In the assembled state disordered regions of the head and tail domains contribute a flexible connection with the rod at the tetrameric interface that can further compress the polymer in its resting state (middle schematic). Under tension stress these interactions are broken and the flexible regions can stretch so that they effectively act as springs that enable reversible dimer compression, stretching and sliding in the assembled lamin A polymer (bottom schematic)

1B) as well as E65-K97 cross-link between adjacent coils 1A and 1B termini in the assembled nucleoskeleton (Fig. 9b asterisk). Similarly, interactions of the unstructured head and tail regions flanking the rod were recapitulated in S12-E33 and E385-S407 cross-links (Fig. 9b double asterisk). Ex vivo cross-links also explain differences between lamins and other intermediate filaments in assembly: lamin dimers uniquely only engage in lateral assembly once incorporated into head-to-tail oligomers ${ }^{14}$. Crosslinking data suggest an antiparallel association of two dimers via their coils 2 (Fig. 9b triple asterisk), with the positively-charged $\mathrm{PH}$ region of one binding the acidic C-terminus of the other (Fig. 9c). In solitary dimers, however, the coil 2 acidic C-terminus is dynamically occupied by the flanking positively-charged unstructured tail region (Figs. 8c, 9d), and thus lateral association with the $\mathrm{PH}$ region would only be possible between head-totail oligomers where tail region interactions shift towards coil 1A (Figs. 8d, e and 9e). This explains earlier experiments where tailless lamin dimer lateral interactions were inhibited by addition of this tail region ${ }^{21}$.

\section{Discussion}

These data indicate that electrostatic interactions between the flanking unstructured head and tail regions with the opposite rod termini of adjacent dimers establish and maintain the head-to-tail interface (Fig. 10). This function of the tail is unique to lamins as they are the only intermediate filaments with positive charge immediately after the rod: this added electrostatic interaction may help direct the order of assembly. By contrast, intermediate filament rod domain structure is more conserved, and its compression through linkers folding flanking coils over one another in a tandem stagger is likely a general property of all intermediate filaments. Strong pulling forces frequently exercised on lamins could break these interactions and extend the rod to increase polymer size via proximal re-organisation of electrostatic and polar interactions and stretching linkers. When force is removed, the unstructured linker and head/tail regions have multiple redundant pathways to re-establish the same compression and assembly endpoints due to the range of possible electrostatic and polar interactions. Thus, linkers and unstructured head/tail regions likely act as springs enabling the stretch and compaction properties of intermediate filaments (Fig. 10). This mechanism can explain many previously inexplicable disease mutations in head/tail/linkers and adjacent coils and, as a strategy to generate elasticity, could be applied in synthetic polymer design.

\section{Methods}

Wild type and mutant lamin A expression vector cloning. Lamin A without tags, its mutants and 6xHis-tagged lamin A were expressed in E.coli using pET28b vector (Novagen, \#69865-3). Sequence encoding human pre-lamin A was PCRamplified to exclude last 18 amino acids (to match the mature lamin A form as processed in a mammalian cell). An internal to lamin A Nco I site at position 1,388 was removed using site-directed mutagenesis and Nco I and BamH I sites were introduced to $3^{\prime}$ and $5^{\prime}$ termini for cloning of untagged wild type and mutant lamin A into pET28b deleating of the N-terminal His-tag. His-tagged lamin A was cloned using $3^{\prime}$ BamH I and 5' Xho I instead. The following de novo (d.n.) lamin A mutants or mutants associated with Emery-Dreifuss Muscular Dystrophy (EDMD), Limb-Girdle Muscular Dystrophy (LGMD1B), Dilated Cardiomyopathy with Conduction Defects (DCM-CD) and Congenital Muscular Dystrophy (CMD) were used in this study: R7/8E double mutant (d.n.), R25G (EDMD/LGMD1B), E33G (EDMD/LGMD1B), E65G (EDMD/LGMD1B), E82K (DCM-CD), R388C (CMD), R388E (d.n.), R401C (EDMD) and R401E (d.n.). Corresponding mutations were introduced into the lamin A coding sequence using site-directed mutagenesis circular PCR of the pET28b-laminA vector. Full list of primers is available in Supplementary Table 2.

SILAC lamin A expression and purification from bacteria. Light and isotopically labelled heavy wild type lamin A were expressed in BL21 DE3 argA lys A cells ${ }^{47}$ grown in $\mathrm{M} 9$ medium supplemented with $0.2 \%$ glucose, $50 \mu \mathrm{g} \mathrm{mL}^{-1}$ kanamycin, $1 \mathrm{mM} \mathrm{MgSO}_{4}$, and either normal or isotopically labelled $\mathrm{L}-{ }^{13} \mathrm{C}_{6}{ }^{15} \mathrm{~N}_{4}$-arginine and $\mathrm{L}-{ }^{13} \mathrm{C}_{6}{ }^{15} \mathrm{~N}_{2}$-lysine $(50 \mu \mathrm{g} \mathrm{mL}-1$ each) (Sigma-Aldrich, \#608033 and \#608041). Overnight cultures were grown and diluted to an $\mathrm{OD}_{600}$ of 0.001 and further grown for additional $8 \mathrm{~h}$ to an $\mathrm{OD}_{600}$ of 0.6 before IPTG induction.

His-tagged lamin A was expressed in conventional BL21 DE3 bacterial cells in LB medium. In either case protein expression was induced with $0.3 \mathrm{mM}$ IPTG for $4 \mathrm{~h}$ and protein was purified from inclusion bodies. Briefly, $1 \mathrm{~L}$ of bacteria were collected by centrifugation at $2,000 \times g$, lysed in $12 \mathrm{ml}$ of $50 \mathrm{mM}$ Hepes, $\mathrm{pH}$ 8.0, $3 \mathrm{mM} \mathrm{MgCl}_{2}, 3 \mathrm{mM}$ 2-mercaptoethanol, $1 \mathrm{mM}$ PMSF, $1 \mu \mathrm{g} \mathrm{mL}^{-1}$ aprotinin, $1 \mu \mathrm{M}$ leupeptin, and $1 \mu \mathrm{M}$ pepstatin by repeated sonication in the presence of DNAseI $\left(1 \mathrm{U}^{-1}\right)$. Inclusion bodies were then pelleted, washed twice in $\mathrm{ddH}_{2} \mathrm{O}$ with $2 \%$ Triton X-100 and resuspended in the buffer with $6 \mathrm{M}$ urea ${ }^{48}$. Protein was equilibrated in either Tris buffer $(25 \mathrm{mM}$ Tris, $250 \mathrm{mM} \mathrm{NaCl}, \mathrm{pH}$ 8.0) or $\mathrm{NaPi}$ buffer (100 mM sodium phosphate, $250 \mathrm{mM} \mathrm{NaCl}, \mathrm{pH}=8.0)$ supplemented with $6 \mathrm{M}$ urea and $1 \mathrm{mM}$ PMSF.

Analytical ultracentrifugation. For analytical ultracentrifugation lamin A was reconstituted immediately after purification in either Tris buffer or $\mathrm{NaPi}$ buffer with $6 \mathrm{M}$ urea. Protein at a concentration of $0.5 \mathrm{mg} \mathrm{mL}^{-1}$ was then dialysed against Tris or NaPi buffer without urea and prepared in $400 \mu \mathrm{l}$ at a final concentration of $0.3 \mathrm{mg} \mathrm{mL}^{-1}$. Sedimentation velocity (SV) experiments were carried out in a Beckman Coulter (Palo Alto, CA, USA) ProteomeLab XL-I analytical ultracentrifuge using interference optics. All AUC runs were carried out at a rotation speed of $45,000 \mathrm{rpm}(154,000 \times g$ at the optical cell centre) and an experimental temperature of $20^{\circ} \mathrm{C}$. The density and viscosity of the Tris buffer and NaPi buffer at the experimental temperature $\left(20^{\circ} \mathrm{C}\right)$ were calculated using program SEDNTERP ${ }^{49}$. The partial specific volume $\left(\bar{v}_{20}\right)$ of protein was calculated as an additive sum of values of constituent amino acids using program SEDNTERP. Sedimentation velocity profiles (separate scans were taken every $4 \mathrm{~min}, 140$ scans in total) were treated using size-distribution $\mathrm{c}(\mathrm{s})$ model implemented in the program SEDFIT $^{50}$. Each peak on the distribution plot was integrated in order to obtain the weight-averaged values for sedimentation coefficient and molecular mass. Integrated values of sedimentation coefficient (s) obtained at experimental conditions were converted to the standard conditions $\left(s_{20, w}\right)$ (which is the value of sedimentation coefficient in water at $20^{\circ} \mathrm{C}$ ).

Sedimentation coefficients $S_{\text {calc }}$ for the lamin A dimeric models with different levels of the rod compression based on cross-linking data and lamin A head-to-tail tetramer mock models were calculated from their atomic coordinates using program SoMo ${ }^{51,52}$.

Lamin homo-iso- and homo-/hetero-iso-dimer mix preparation. Homo-isodimer mix of light and heavy lamin A was prepared by mixing light and isotopiocally labelled heavy lamin $\mathrm{A}$ at a 1:1 ratio in $\mathrm{NaPi}$ buffer in which we have shown that lamin dimers do not exchange chains (Supplementary Fig. 2).

Homo-/hetero-iso-dimer mix was prepared by running the homo-iso-dimer mix on a preparative Hoefer $180 \mathrm{~mm} \times 160 \mathrm{~mm} \mathrm{10 \%} \mathrm{Laemmli} \mathrm{SDS-PAGE} \mathrm{gel.} \mathrm{A}$ 
total of $1.6 \mathrm{mg}$ of protein per a single slab was run for $8 \mathrm{~h}$ at $150 \mathrm{~V}$. The area containing monomerised and mixed-in-the-gel light and heavy lamin $\mathrm{A}$ was identified via IntstantBlue (Expedeon, \#ISB1L) staining of a thin strip of the gel Unstained protein was then gel-extracted and desalted 28,29 via precipitation with $400 \mathrm{mM} \mathrm{KCl}$ and sequential washes with an 86:7:7 (vol:vol) mix of Acetone, Triethylamine and Acetic Acid and the same solution diluted to $5 \%$ in $\mathrm{ddH}_{2} \mathrm{O}^{48}$; then and reconstituted in $\mathrm{NaPi}$ buffer with $6 \mathrm{M}$ urea. Successful refolding of gelextracted lamin A was confirmed by rotary metal shadowing EM. As light and heavy lamin A dimers were monomerised together on an SDS-PAGE in this procedure, refolded protein sample should contain a combinatorial mix of $25 \%$ light-light, 25\% heavy-heavy and 50\% light-heavy lamin A dimers.

Lamin A in vitro cross-linking and mass spectrometry. Cross-linking of lamin A in vitro was carried out at a protein concentration of $0.4 \mathrm{mg} \mathrm{mL}^{-1}$ in NaPi buffer: Homo-iso-dimer or homo-/hetero-iso-dimer lamin A mixes were dialysed into $\mathrm{NaPi}$ buffer at a concentration of $0.5 \mathrm{mg} \mathrm{mL}^{-1}$ and adjusted to $0.4 \mathrm{mg} \mathrm{mL}^{-1}$ in each sample. This concentration was chosen as one close to maximum concentration at which lamin A can stay soluble in the chosen buffer for the duration of the cross-linking experiment.

Samples prepared in this manner were then cross-linked with a mixture of EDC (1-ethyl-3-(3-dimethylaminopropyl)carbodiimide hydrochloride) and Sulfo-NHS (N-hydroxysulfosuccinimide) (Thermo Fisher Scientific Pierce, \#PG82074 and \# 24510) at a weight ratio of 1:4:8.8 (lamin A:EDC:S-NHS) for $30 \mathrm{~min}$ at room temperature. The reaction was then quenched for $10 \mathrm{~min}$ by addition of $1 \mathrm{M}$ Tris at $\mathrm{pH} 8.0$ to a final concentration of $50 \mathrm{mM}$. For SDS-PAGE analysis the quenched cross-linking reaction was split in two halves and resolved on a preparative poly acrylamide gels: $7.5 \%$ Bis-Tris gel for mass spectrometry analysis, or a $7.5 \%$ Laemmli gel for gel-extraction of individual bands.

For MS analysis, polyacrylamide gels with resolved cross-linking reaction product-bands were stained with InstantBlue and de-stained with $\mathrm{ddH}_{2} \mathrm{O}$ before band excision.

The bands corresponding to cross-linked complexes were excised and the proteins therein were reduced using $10 \mathrm{mM}$ DTT for $30 \mathrm{~min}$ at room temperature, alkylated with $55 \mathrm{mM}$ iodoacetamide for $20 \mathrm{~min}$ in the dark at room temperature and digested using $13 \mathrm{ng} \mu \mathrm{L}^{-1}$ trypsin (Thermo Fisher Scientific) overnight at $37^{\circ}$ $\mathrm{C}^{53}$ and digested peptides were fractionated using SCX-Stage-Tips ${ }^{54}$. In short, peptide mixtures were first loaded on a SCX-Stage-Tip in $0.5 \%(\mathrm{v} / \mathrm{v})$ acetic acid $20 \%(\mathrm{v} / \mathrm{v})$ acetonitrile, $50 \mathrm{mM}$ ammonium acetate and sequentially eluted with buffers containing $100 \mathrm{mM}$ ammonium acetate and $500 \mathrm{mM}$ ammonium acetate (two fractions each). Each peptide fraction was then desalted using C18-StageTips ${ }^{55,56}$ prior to mass spectrometric analysis.

LC-MS/MS analysis of peptides in the in vitro cross-linking experiments was performed on an LTQ Orbitrap Velos mass spectrometer (Thermo Fisher Scientific) that was coupled with a Dionex Ultimate 3000RSLC nano HPLC system, using a high/high strategy ${ }^{57}$, both MS spectra and MS2 spectra were acquired in the Orbitrap. The analytical column with a self-assembled particle frit ${ }^{54}$ and C18 material (ReproSil-Pur C18-AQ $3 \mu \mathrm{m}$; Dr. Maisch, $\mathrm{GmbH}$ ) was packed into a spray emitter (75- $\mu \mathrm{m} \mathrm{ID,} \mathrm{8- \mu m} \mathrm{opening,} \mathrm{300-mm} \mathrm{length;} \mathrm{New} \mathrm{Objective)} \mathrm{using} \mathrm{an} \mathrm{air-}$ pressure pump (Proxeon Biosystems). Mobile phase A consisted of water with $0.1 \%$ formic acid. Mobile phase B consisted of $80 \%$ acetonitrile with $0.1 \%$ formic acid.

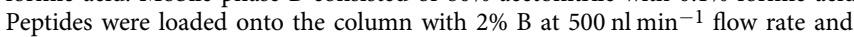
eluted at $200 \mathrm{nl} \mathrm{min}^{-1}$ flow rate, with a linear gradient increased from 2 to $40 \%$ acetonitrile in $0.1 \%$ formic acid in $139 \mathrm{~min}$ and then an increase from 40 to $95 \% \mathrm{~B}$ in $11 \mathrm{~min}$. Mass spectra were recorded at 100,000 resolution. The eight highest intensity peaks with a charge state of three or higher were selected in each cycle for ion-trap fragmentation. The fragments were produced using collision-induced dissociation (CID) with $35 \%$ normalised collision energy and detected by the Orbitrap at 7500 resolution. Dynamic exclusion was set to single repeat count and $90 \mathrm{~s}$ exclusion duration. The mass spectrometric raw data were processed to generate peak lists by MaxQuant (Version 1.5.3.30) ${ }^{58}$ and cross-linked peptides were matched to spectra using Xi software (version 1.6.745) ${ }^{59}$ with in-search assignment of monoisotopic peaks ${ }^{60}$ and the following parameters: sequence database human LaminA; cross-linker: EDC; MS accuracy, 6 ppm; MS/MS accuracy, 20 ppm; enzyme: trypsin; missed cleavages, 4; missing mono-isotopic peaks: 4; fixed modification: carbamidomethylation on cysteine; variable modifications: oxidation on methionine; R10 and K8 for SILAC samples. Search database is human lamin A with decoy setting. FDR was estimated using XiFDR (version 1.1 .27 ) on $5 \%$ residue level ${ }^{61}$.

\section{Lamin A ex vivo cross-linking and mass spectrometry. Rat liver nuclear} envelopes were purified according to standard procedures developed in Schirmer $\mathrm{lab}^{62}$. Livers were obtained from Sprague Dawley rats provided by the University of Edinburgh animal facility in compliance with local ethics and Home Office procedures. Liver tissue was homogenised and nuclei were isolated and stripped of the remaining endoplasmic reticulum and contaminants in a series of centrifugation spins through sucrose cushions of increasing concentrations. Intact chromatin still inside the nuclei was then digested with DNAse and micrococcal nuclease (MNAse) and washed away from the nuclei in a hypotonic buffer as confirmed by means of Hoechst staining that revealed only a thin rim of chromatin still clinging to the nucleoskeleton. Purified nuclear envelopes were then pelleted and equilibrated in NaPi buffer and cross-linked with a 1:2.2 mixture of EDC/S-NHS supplied in twofold and fourfold NE protein weight excess for $30 \mathrm{~min}$. Reactions were quenched with $50 \mathrm{mM}$ Tris $\mathrm{pH} 8.0$ for $10 \mathrm{~min}$. Optionally purified nuclear envelopes were additionally extracted with $0.5 \%$ Triton X-100 and $400 \mathrm{mM} \mathrm{KCl}$ to obtain lamina-pore complex nuclear envelope shells ${ }^{63}$.

Successful nucleoskeletal lamin A cross-linking was confirmed by Western blotting of cross-linked nuclear envelopes lysed in NuPage LDS buffer (Thermo Fisher Scientific) and resolved on an 8\% Bis-Tris polyacrylamide gel with the 5881 rabbit polyclonal antibody ${ }^{64}$ against a region [572-585] downstream of the Ig fold in the lamin A tail domain (at a 1:250 dilution). LICOR anti-rabbit IR800 antibody (Li-Cor Biosciences, \#925-32213, 1:2,500 dilution) was used as secondary. Imaging was done with a Li-Cor Odyssey CLx imaging system (Li-Cor Biosciences).

In preparation for MS analysis cross-linked nuclear envelopes were washed in $1 \mathrm{M} \mathrm{NaCl}$ and the lamina was solubilised in $0.1 \mathrm{M}$ Tris $8.5,4 \mathrm{M}$ urea, $20 \mathrm{mM}$ $\mathrm{MgCl}_{2}$. Around $10 \%$ of total nuclear envelope protein was recovered this way and then processed for mass spectrometry by means of sequential overnight digestion with endoproteinase Lys-C (Roche, \# 11058533103) dilution to $2 \mathrm{M}$ urea and overnight digestion with Trypsin (Thermo Fisher Scientific, \# 90057). Alternatively in-gel $^{53}$ digestion and FASP digestion ${ }^{65}$ was used. In either case digested peptides were fractionated using SCX-Stage-Tips as described in the in vitro cross-linking method section.

LC-MS/MS analysis of peptides in ex vivo cross-linking experiments was performed using either an LTQ Orbitrap Velos mass spectrometer (Thermo Fisher Scientific, details see above) or an Orbitrap Fusion ${ }^{\text {mex }}$ Lumos $^{\text {tw }}$ Tribrid $^{\text {ot }}$ Mass Spectrometer (Thermo Scientific) (as specified in the raw MS data files) applying a "high-high" acquisition strategy. Peptides were separated on a $75 \mu \mathrm{m} \times 50 \mathrm{~cm}$ PepMap EASY-Spray column (Thermo Scientific) fitted into an EASY-Spray source (Thermo Scientific), operated at $50^{\circ} \mathrm{C}$ column temperature. The eluted peptides were directly introduced into the mass spectrometer. MS data were acquired in the data-dependent mode with the top-speed option. For each three-second acquisition cycle, the survey level spectrum was recorded in the Orbitrap with a resolution of 120,000 . The ions with a precursor charge state between $3+$ and $8+$ were isolated and fragmented using high energy collision dissociation (HCD) of 30\% normalised collision energy. The fragmentation spectra were recorded in the Orbitrap with a resolution of 15,000 , isolation window of $1.6 \mathrm{~m} / z$, maxium injection time of $60 \mathrm{~ms}$ and AGC target of 5e4. Dynamic exclusion was enabled with single repeat count and $60 \mathrm{~s}$ exclusion duration. The mass spectrometric raw data were processed to generate peak lists by MSCovert (ProteoWizard 3.0.11417) ${ }^{66}$ and cross-linked peptides were matched to spectra using Xi software (version 1.6.745) ${ }^{59}$. The parameters are the same as for in vitro data analysis except for using a database of rat lamin A instead of human. The data has been manually validated.

All the mass spectrometry proteomics data have been deposited to the ProteomeXchange Consortium via the PRIDE ${ }^{67}$ partner repository with the dataset identifier PXD008337 and PXD014009.

SILAC cross-linking data analysis. A total of 1,382 cross-link spectra were identified in dimer-rich bands $1-3$ in the homo-iso-dimer mix (HIDm X) experiment after FDR using XiSearch engine (version 1.6.745) ${ }^{59}$ and XiFDR (v 1.1.27) ${ }^{61}$. Similarly 1.322 cross-link spectra were identified in dimer-rich bands $1-3$ in the homo-/hetero-iso-dimer experiment (H/hIDm X). Identified spectra of peptide pairs with incomplete SILAC labelling were discarded. To increase the stringency of our analysis only spectra with match score below 8.0 were also discarded, leaving 807 and 858 spectra (1,665 total) supporting 387 and 365 unique cross-linked residue pairs in the respective cross-linking experiments. These two sets overlapped in 243 unique cross-linked residue pairs-cross-links identified in both experiments. These were supported by 1308 spectra out of total $1665(78 \%)$ attesting to a high reproducibility of abundant cross-links.

To determine the inter-/intra-dimeric and inter-/intra-chain origin of each of the 243 cross-links a comparative cross-linking analysis ${ }^{30,31}$ routine was adapted (Supplementary Discussion): for each of the 1.308 spectra relative ion intensities in four peptide-pair precursor ion clusters-light-light peptide-pair ion cluster (LL), heavy-light (HL), light-heavy (LH) and heavy-heavy (Fig. 2e-g) - were identified and quantified across the pertinent elution peak (extracted ion chromatograms XiCs) containing this spectra MS and MS:MS. Due to the extreme complexity of the sample these were quantified manually in Thermo Xcalibur software (Thermo Fisher Scientific 4.0.27.1) as average peak intensities across pertinent XiCs. For a select set of spectra $\mathrm{XiC}$ areas were also quantified semi-automatically using Skyline $\mathrm{e}^{32,68}$ (v 3.7.0.11317). In each case mono-, first and second isotopic peak $\mathrm{XiCs}$ were analysed in each of 4 peptide ion clusters. Single XiC was used for all peaks in Xcalibur and individual XiCs were aligned manually in Skyline. Relative $\mathrm{HL}$ and $\mathrm{LH}$ peptide pair ion abundances were quantified as ratios $(\mathrm{HL}+\mathrm{LH}) /$ $(\mathrm{LL}+\mathrm{HL}+\mathrm{LH}+\mathrm{HH})$ for each spectra. Then frequencies of cross-link occurrence between differentially labelled peptides were calculated using as median values of $(\mathrm{HL}+\mathrm{LH}) /(\mathrm{LL}+\mathrm{HL}+\mathrm{LH}+\mathrm{HH})$ ratios of all spectra supporting this cross-link in HIDm X and H/hIDm X experiments separately (Supplementary Data 4). Ratios calculated using Xcalibur and Skyline appeared to match closely and lay within 6\% of each other for $95 \%$ of measurements (Supplementary Data 1-3). These were converted into frequencies $\mathrm{F}^{\text {Inter-dimeric }} \mathrm{F}^{\text {Inter-molecular-of cross-link occurrence }}$ respectively as inter-dimeric in the HIDm X experiment and as inter-chain in the H/hIDm X experiment (Fig. 1g). Respective absolute frequencies of cross-link 
occurrence as intra-dimeric-inter-chain ( $\left.\mathrm{F}^{\text {Inter-chain }}\right)$ and intra-chain ( $\left.\mathrm{F}^{\text {Intra-chain }}\right)$ were then calculated as $F^{\text {Inter-chain }}=F^{\text {Inter-molecular }} \mathrm{F}^{\text {Inter-dimeric }}$ and $\mathrm{F}^{\text {Intra- }}$ chain $=1-F^{\text {Inter-chain }}-\mathrm{F}^{\text {Inter-dimeric }}=1-\mathrm{F}^{\text {Inter-molecular }}$. A number of cross-links have shown a propensity to happen both between and within dimers and both between and within chains owning to two factors: on the one hand, the extreme flexibility and assembly/aggregation tendency of lamins; and on the other, the fact that majority residues in the rod domain can be considered as surface residues and thus can engage in both intra- and inter-molecular interactions. For each cross-linked region of lamin $\mathrm{A}$ we aimed to reconstruct its frequent conformation(s). To this end during consequent interrogation of the obtained cross-links for structural information the following arbitrary interpretation of the $F^{\text {Inter-dimeric }}, F^{\text {Inter-chain }}$ and FIntra-chain were in effect: a cross-link with the $F^{\text {Inter-dimeric }} \geq 80 \%$ was considered to be mostly inter-dimeric and not considered for lamin A dimer structure; within the remaining pool of cross-link each was then considered intrachain in a dimer if the $\mathrm{F}^{\text {Inter-chain }} /\left(\mathrm{F}^{\text {Intra-chain }}+\mathrm{F}^{\text {Inter-chain }}\right)<20 \%$ (among all dimers containing this cross-link, in $80 \%$ or more cases this cross-link happens within a single chain); considered inter-chain in a dimer if $\mathrm{F}^{\text {Inter-chain }} / \mathrm{F}^{\text {Intra-chain }}+$ FInter-chain) $\geq 80 \%$ (happens between the two chains in $80 \%$ or more cases); considered to happen both ways-intra-chain and inter-chain in a dimer if $F^{\text {Inter- }}$ chain $/\left(F^{\text {Intra-chain }}+\mathrm{F}^{\text {Inter-chain }}\right)$ was between 20 and $80 \%$.

Additionally, 220 spectra supporting 143 unique cross-linked residue pairs were identified in the tetramer-rich band 4 in the homo-iso-dimer mix experiment. These were similarly processed to obtain ( $\left.F^{\text {Inter-dimeric }}\right)$.

Based on this information it was possible to assess fitness of the cross-links to the prediction based model of lamin A.

Ex vivo cross-linking data analysis. Thirty nine spectra supporting 15 unique cross-linked residue pairs were identified from multiple ex vivo cross-linking experiments. Two cross-links-E31-K32 and S143-E145-were unambiguously identified as inter-molecular as these occurred between pairs of overlapping peptides. Remaining cross-links were presumed as inter- or intra-molecular based on in vitro data.

Cross-linking data visualisation. Cross-linking data from all experiments were visualised using xiNET (http://crosslinkviewer.org/index.php) ${ }^{69}$.

Cross-linked protein isolation via gel-extraction. To be able to relate crosslinking patterns obtained from each cross-linking product-band to the lamin A oligomeric architecture in the same product-band the second half of the reaction was resolved on 7.5\% Laemmli SDS-PAGE. Upon resolution a thin band was cut to identify the exact migration pattern and individual bands were excised, gelextracted, desalted and reconstituted as described before for the homo-/hetero-isodimer lamin A mix.

Rotary metal shadowing EM. For rotary metal shadowing EM of un-cross-linked lamin A, the protein was equilibrated in either Tris buffer or NaPi buffer at a concentration of $0.1 \mathrm{mg} \mathrm{mL}^{-1}$ by dialysis against 1,000 volumes twice. For rotary metal shadowing of cross-linked lamin A and material in the individual crosslinked bands, protein in each sample was equilibrated in NaPi buffer at the same concentration again by dialysis. Glycerol was then added to the equilibrated lamin A samples to a final concentration of $30 \%$ and samples were sprayed onto roughly square $10 \times 10 \mathrm{~mm}$ mica sheet pieces (TAAB, \#M460) using a glycerol spraying device $^{26}$ graciously provided by Prof. Ueli Aebi. These were then rotary metal shadowed in an ACE600 Leica vacuum evaporator at a pressure of $1.0-2.5 \times 10^{-5}$ mbar with $2 \mathrm{~nm}$ of platinum and $9 \mathrm{~nm}$ of carbon at respectively $6^{\circ}$ and $90^{\circ}$ elevation angles. Coated mica sheet pieces were then incubated in a moist chamber at $60^{\circ} \mathrm{C}$ for $30 \mathrm{~min}$ and platinum/carbon cast films were floated on water. Pieces of these films were picked with EM copper grids and air-dried. Imaging was performed using JEOL 1200 TEM at $80 \mathrm{kV}$ and magnification of $\times 20,000$. Micrographs were then analysed in ImageJ and lamin A rod length was recorded. Distributions were not assumed normal, and non-parametric Kruskal-Wallis test followed by pair-wise comparison Dunn test with Holm correction were carried out to estimate shift in rod length distributions.

Basic lamin A dimer modelling. Basic model of lamin A dimer with $51 \mathrm{~nm}$ long rod was built using existing structures, homology modelling and de novo modelling. Coil 1A, part of L1 and the bulk of coil 1B were modelled de novo using CCbuilder (v 1.0) ${ }^{70}$. Residues [181-220] in the C-terminus of coil 1B were modelled by homology with vimentin: 3UF1 [https://doi.org/10.2210/pdb3UF1/pdb] structure $^{34}$ fragment containing residues [208-247] was used as a template for SWISS-MODEL (https://swissmodel.expasy.org//71-74. Similarly, the N-terminal half of coil 2 containing a parallel hendecad (PH) residues [240-277] and coil 2 residues [288-310] were modelled by homology with vimentin using 3TRT [https://doi.org/10.2210/pdb3TRT/pdb] structure ${ }^{35}$ fragment containing residues [264-334] as a template for SWISS-MODEL. The C-terminal half of coil 2residues [313-386] - was directly copied from $1 \times 8 \mathrm{Y}$ [https://doi.org/10.2210/ pdb1X8Y/pdb] crystal structure ${ }^{8}$. Missing residues in the rod were built using modelled fragments as a single template for SWISS-MODEL. N-terminal head and the tail domains were modelled using iTasser $^{75-77}$. Residues of the Ig fold were then substituted by superposition of the 1IFR [https://doi.org/10.2210/pdb1IFR/ $\mathrm{pdb}]$ crystal structure ${ }^{10}$. All further manipulation of the disordered regions to build projection models in Fig. 8 and Supplementary Figs. 7 and 9 were done in PyMol$^{78}$.

Molecular modelling of the lamin A head-to-tail tetramers. To calculate theoretical sedimentations coefficient $\mathrm{S}_{\text {calc }}$ for lamin A head-to-tail tetramers, mock tetrameric models were created using Rosie docking2 protocol-part of the Rosetta online public server ${ }^{79-81}$. Coil 2 fragment [354-385] was roughly aligned to coil 1 A fragment [28-67] in a half-staggered manner as an input for docking2 to produce models with 2-3-heptad-long interfaces thought to occur in during lamin head-to-tail tetramerisation ${ }^{24}$. Alternatively longer fragments [320-385] and [28-90] were used to produce models with interface spanning the entire length of coil 1A. Among top 10 scoring models those with roughly parallel coiled coil fragment alignment were selected and rest of the dimers' rod and unstructured region were build using the basic lamin A dimer model (see section on Basic lamin A dimer modelling) in PyMol.

Molecular modelling of the rod coiled coil segment overlap. To model lamin A coiled coil termini overlap and rod compression from available coil-to-coil crosslinks across linkers L1, L12, L2 and L3 cross-link guided molecular modelling was employed $^{43,82}$ that utilises ROSETTA (build 2017.08.59291) global docking protocols $^{83}$ and Xwalk (v 0.6$)^{44}$ to select structures satisfying input cross-links. Rosetta and Xwalk command parameters are detailed in Supplementary Note 1.

Here linkers were assumed flexible and thus only cross-links between coiled coil termini were used as an input (Supplementary Data 5 and 6). Intra- and intermolecular cross-links were used in duplicates (i.e. respectively from chain A to chain A and from chain B to chain B; from chain A to chain B and from chain B to chain $A)$, cross-links that happen both ways were used in tetraplets $(A-A, A-B$, B-A, B-B) Docking was performed between dimeric coiled coil fragments [46-66] and [78-102] separated by L1, [201-219] and [240-260] sepparated by L12, [241-254] and [267-291] assumed separated by (L2), and [256-276] and [289-310] assumed separated (L3). A total of 100,000 models were generated for each pair of fragments.

Models with negative I_sc (Rosetta's Interface score calculated as a difference between the total energy of the complex and the sum of total energies of each partner in isolation; models with I_sc $<-5.0$ are considered as good decoys as detailed in RosettaDock application documentation https://www.rosettacommons. org/docs) were chosen and surface accessible distances $\left(\mathrm{C}_{\beta}-\mathrm{C}_{\beta}\right.$ SAS distances) between pairs of $\mathrm{C}_{\beta}$ atoms of cross-linked residues were calculated with Xwalk (-xSC option) and in some cases manually (Supplementary Note 1).

Models failing to satisfy any cross-links were discarded. Angles between docked dimeric fragments were calculated in PyMol using angle_between_helices function from psico module (by Thomas Holder and Steffen Schmidt, https://github.com/ speleo3/pymol-psico). Of the remaining models only those with two dimeric fragments in parallel or close to parallel orientation (tandem stagger models) were picked for further analysis, while the rest (anti-parallel folds) were discarded. Tandem stagger models were additionally refined using Rosetta local refinement protocols to achieve better interface scores. Cross-links were re-validated with Xwalk and tandem stagger structures with negative I_sc, satisfying cross-links were included into a final set (Supplementary Data 7-11). Rod shortening conveyed by each model was then calculated in PyMol as change in distance between the straight dimer rod length and the rod length in each model, calculated as a sum of rod fragments before and after the tandem stagger point and thus taking in consideration the bending of the rod introduced in the stagger. Finally models with I_sc $\leq-5.0$ were deemed as those with stable interface between docked dimeric fragments.

In further search for a more exhaustive list of tandem stagger models Rosetta was run in an unconstrained mode-with no cross-links as an input-for each pair of dimeric coiled coil fragments separated by linker L1, L12, (L2) and (L3). Models with negative I_sc were initially filtered with Xwalk for those roughly satisfying cross-links (as defined by maximal $\mathrm{C}_{\beta}-\mathrm{C}_{\beta}$ SAS distances for cross-linked residues of $13 \AA$ ). Any models potentially satisfying at least one cross-link were then refined and models with I_sc above -5.0 were discarded. Remaining models were filtered for compliance with maximal extension allowed by the respective linkers L1, L2 and L3 (SAS $46 \AA$ S66O-K78N, SAS $46 \AA$ D254O-Y267N and SAS $46 \AA$ Q276O$\mathrm{H} 289 \mathrm{~N})$ via Xwalk. The linker L12 is sufficiently long to accommodate any degree of overlap dictated by coil 1B-to-PH cross-links and is altogether longer than maximal SAS distance of $60 \AA$ that Xwalk can calculate; therefore filtering for compliance with L12 extension was redundant. Remaining models were then subject to a more stringent test for cross-link satisfaction via Xwalk in search for models where cross-linked residues already engage in electrostatic or polar interaction with each other. To this end instead of $\mathrm{C}_{\beta}-\mathrm{C}_{\beta}$ SAS distances straight line Euclidean distances were calculated between carboxyl-group oxygen atoms and side chain amine group nitrogen atoms (for lysine-involving cross-links) or hydroxyl group oxygen atoms (for serine, tyrosine or threonine involving crosslinks). Residues were then assumed cross-linkable if these distances did not exceed $4 \AA$ and thus satisfying close range electrostatic interaction criteria of $4 \AA$ between oxygen and nitrogen atoms in COO- and $\mathrm{CH}_{2} \mathrm{NH}_{3}+\left(\right.$ for D/E-K) ${ }^{84}$ or hydrogen 
bond criteria (for $\mathrm{D} / \mathrm{E}-\mathrm{Y} / \mathrm{T} / \mathrm{S})^{85}$. Angles between docked fragments were calculated in PyMol and tandem stagger models were selected.

Linker regions reconstruction for hydrodynamic calculations. To carry out hydrodynamic calculations and determine sedimentation coefficients of lamin A rod in various stages of compression, docked dimeric fragments from Z-fold models were incorporated into the basic lamin A dimer model and missing linker regions were reconstructed using SWISS-MODEL or MODELLER ${ }^{86}$. Input commands for the latter are detailed in Supplementary Note 1.

Electrostatic potential surface (EPS) reconstruction. EPS reconstructions were carried out for coil 1A, coil $2 \mathrm{C}$-terminus and head and part of the tail before the Ig domain using PDB2PQR (v 2.1.1) servers (http://nbcr-222.ucsd.edu/ pdb2pqr_2.1.1/ $)^{87}$ in Amber force field at pH 8.0. Output.pqr files were then computed in APBS using linearized Poisson-Boltzmann Equation; mobile ions were set to match salt concentration in the cross-linking buffer. All relevant Figures feature EPS with 50\% transparency as visualised in PyMol.

In vitro assembly assays. Reconstituted lamin $\mathrm{A}$ and its mutants were first equilibrated in $25 \mathrm{mM}$ Tris, $8 \mathrm{M}$ urea, $250 \mathrm{mM} \mathrm{NaCl}, \mathrm{pH} 8.8$ at a concentration of $0.3 \mathrm{mg} \mathrm{mL}^{-1}$ and then dialysed out of urea against $25 \mathrm{mM}$ Tris, $250 \mathrm{mM} \mathrm{NaCl}, 1$ $\mathrm{mM}$ DTT, $1 \mathrm{mM}$ EGTA, pH 8.5 for $3 \mathrm{~h}$ and then against $25 \mathrm{mM}$ MES, $150 \mathrm{mM}$ $\mathrm{NaCl}, 1 \mathrm{mM}$ DTT, $1 \mathrm{mM}$ EGTA pH 6.5 for an additional $30 \mathrm{~min}$. For the general solubility assessment, samples were then centrifuged at $100,000 \times g$ for $30 \mathrm{~min}$, supernatant was immediately removed as soluble phase (S) and an equal volume of $25 \mathrm{mM}$ Tris, $8 \mathrm{M}$ urea, $250 \mathrm{mM} \mathrm{NaCl}$, pH 8.8 was added to the centrifuge tubes to solubilise pelleted lamin A oligomers (P). The experiment was carried out in biological triplicate, normal distribution was assumed and statistical significance was estimated in T-test comparison of mutant samples to the control sample. For a more detailed assessment of lamin A head-to-tail oligomerisation after dialysis, samples were supplemented with glycerol to a final concentration of $30 \%$ and processed for rotary metal shadowing as described above. Lamin A dimers were then scored as singular or incorporated into head-to-tail oligomers in ImageJ.

Reporting summary. Further information on research design is available in the Nature Research Reporting Summary linked to this article.

\section{Data availability}

All the mass spectrometry proteomics data generated in this study have been deposited to the ProteomeXchange Consortium via the $\mathrm{PRIDE}^{67}$ partner repository with the dataset identifiers PXD008337 and PXD014009. Rosetta molecular modelling data is available via Edinburgh DataShare (https://datashare.is.ed.ac.uk/handle/10283/3348). The source data underlying Figs. 1c-f, 2a, c, 7c, 8h, i, 9a and Supplementary Figs. 1a, b, 2a, and 6 are provided as a Source Data file. All other data supporting the findings of this study are available from the corresponding authors upon reasonable request.

Received: 19 September 2018 Accepted: 20 June 2019

Published online: 11 July 2019

\section{References}

1. Worman, H. J. Nuclear lamins and laminopathies. J. Pathol. 226, 316-325 (2012).

2. Swift, J. et al. Nuclear lamin-A scales with tissue stiffness and enhances matrix-directed differentiation. Science 341, 1240104 (2013).

3. Harada, T. et al. Nuclear lamin stiffness is a barrier to $3 \mathrm{D}$ migration, but softness can limit survival. J. Cell Biol. 204, 669-682 (2014).

4. Denais, C. M. et al. Nuclear envelope rupture and repair during cancer cell migration. Science 352, 353-358 (2016).

5. Aebi, U., Cohn, J., Buhle, L. \& Gerace, L. The nuclear lamina is a meshwork of intermediate-type filaments. Nature 323, 560-564 (1986).

6. Heitlinger, E. et al. Expression of chicken lamin B2 in Escherichia coli: characterization of its structure, assembly, and molecular interactions. J. Cell Biol. 113, 485-495 (1991).

7. Turgay, Y. et al. The molecular architecture of lamins in somatic cells. Nature 543, 261-264 (2017).

8. Strelkov, S. V., Schumacher, J., Burkhard, P., Aebi, U. \& Herrmann, H. Crystal structure of the human lamin A coil 2B dimer: implications for the head-totail association of nuclear lamins. J. Mol. Biol. 343, 1067-1080 (2004).

9. Krimm, I. et al. The Ig-like structure of the C-terminal domain of lamin A/C, mutated in muscular dystrophies, cardiomyopathy, and partial lipodystrophy. Structure 10, 811-823 (2002).

10. Dhe-Paganon, S., Werner, E. D., Chi, Y.-I. \& Shoelson, S. E. Structure of the globular tail of nuclear lamin. J. Biol. Chem. 277, 17381-17384 (2002).
11. Kapinos, L. E., Burkhard, P., Herrmann, H., Aebi, U. \& Strelkov, S. V. Simultaneous formation of right- and left-handed anti-parallel coiled-coil interfaces by a coil2 fragment of human lamin A. J. Mol. Biol. 408, 135-146 (2011).

12. Janmey, P. A., Euteneuer, U., Traub, P. \& Schliwa, M. Viscoelastic properties of vimentin compared with other filamentous biopolymer networks. J. Cell Biol. 113, 155-160 (1991).

13. Herrmann, H. \& Strelkov, S. V. History and phylogeny of intermediate filaments: now in insects. BMC Biol. 9, 16 (2011).

14. Herrmann, H. \& Aebi, U. Intermediate filaments: molecular structure, assembly mechanism, and integration into functionally distinct intracellular scaffolds. Annu. Rev. Biochem. 73, 749-789 (2004).

15. Herrmann H. \& Aebi U. Intermediate filaments: structure and assembly. Cold Spring Harb. Perspect. Biol. 8, a018242 (2016).

16. Weber, K., Plessmann, U. \& Ulrich, W. Cytoplasmic intermediate filament proteins of invertebrates are closer to nuclear lamins than are vertebrate intermediate filament proteins; sequence characterization of two muscle proteins of a nematode. Embo J. 8, 3221-3227 (1989).

17. Erber, A. et al. Characterization of the Hydra lamin and its gene: a molecular phylogeny of metazoan lamins. J. Mol. Evol. 49, 260-271 (1999).

18. Guzenko, D., Chernyatina, A. A. \& Strelkov, S. V. Crystallographic studies of intermediate filament proteins. Subcell. Biochem. 82, 151-170 (2017).

19. Chernyatina, A. A., Hess, J. F., Guzenko, D., Voss, J. C. \& Strelkov, S. V. How to study intermediate filaments in atomic detail. Methods Enzym. 568, 3-33 (2016).

20. Stuurman, N., Heins, S. \& Aebi, U. Nuclear lamins: their structure, assembly, and interactions. J. Struct. Biol. 122, 42-66 (1998).

21. Sasse, B., Aebi, U. \& Stuurman, N. A tailless Drosophila lamin Dm0 fragment reveals lateral associations of dimers. J. Struct. Biol. 123, 56-66 (1998).

22. Qin, Z., Kreplak, L. \& Buehler, M. J. Hierarchical structure controls nanomechanical properties of vimentin intermediate filaments. PLoS ONE $\mathbf{4}$, e7294 (2009).

23. Heitlinger, E. et al. The role of the head and tail domain in lamin structure and assembly: analysis of bacterially expressed chicken lamin A and truncated B2 lamins. J. Struct. Biol. 108, 74-89 (1992).

24. Kapinos, L. E. et al. Characterization of the head-to-tail overlap complexes formed by human lamin A, B1 and B2 "half-minilamin" dimers. J. Mol. Biol. 396, 719-731 (2010).

25. Moir, R. D., Donaldson, A. D. \& Stewart, M. Expression in Escherichia coli of human lamins A and C: influence of head and tail domains on assembly properties and paracrystal formation. J. Cell Sci. 99, 363-372 (1991).

26. Aebi U., Baschong W. in Cell Biology 3rd edn (ed. Celis, J. E.) 241-246 (Academic Press, Cambridge, MA, 2006).

27. Schirmer, E. C., Homann, O. R., Kowal, A. S. \& Lindquist, S. Dominant gainof-function mutations in Hsp104p reveal crucial roles for the middle region. Mol. Biol. Cell 15, 2061-2072 (2004).

28. Steinert, P. M., Marekov, L. N., Fraser, R. D. \& Parry, D. A. Keratin intermediate filament structure. Crosslinking studies yield quantitative information on molecular dimensions and mechanism of assembly. J. Mol. Biol. 230, 436-452 (1993).

29. Steinert, P. M., Idler, W. W., Cabral, F., Gottesman, M. M. \& Goldman, R. D. In vitro assembly of homopolymer and copolymer filaments from intermediate filament subunits of muscle and fibroblastic cells. Proc. Natl Acad. Sci. USA. 78, 3692-3696 (1981).

30. Schmidt, C. et al. Comparative cross-linking and mass spectrometry of an intact F-type ATPase suggest a role for phosphorylation. Nat. Commun. 4 1985 (2013)

31. Schmidt, C. \& Robinson, C. V. A comparative cross-linking strategy to probe conformational changes in protein complexes. Nat. Protoc. 9, 2224-2236 (2014).

32. Chen, Z. A. \& Rappsilber, J. Quantitative cross-linking/mass spectrometry to elucidate structural changes in proteins and their complexes. Nat. Protoc. 14, 171-201 (2019)

33. Hess, J. F., Budamagunta, M. S., Shipman, R. L., FitzGerald, P. G. \& Voss, J. C. Characterization of the linker 2 region in human vimentin using site-directed spin labeling and electron paramagnetic resonance. Biochemistry 45, 11737-11743 (2006).

34. Aziz, A. et al. The structure of vimentin linker 1 and rod $1 \mathrm{~B}$ domains characterized by site-directed spin-labeling electron paramagnetic resonance (SDSL-EPR) and X-ray crystallography. J. Biol. Chem. 287, 28349-28361 (2012).

35. Chernyatina, A. A. \& Strelkov, S. V. Stabilization of vimentin coil2 fragment via an engineered disulfide. J. Struct. Biol. 177, 46-53 (2012).

36. Parry, D. A. Hendecad repeat in segment $2 \mathrm{~A}$ and linker L2 of intermediate filament chains implies the possibility of a right-handed coiled-coil structure. J. Struct. Biol. 155, 370-374 (2006). 
37. Chernyatina, A. A., Nicolet, S., Aebi, U., Herrmann, H. \& Strelkov, S. V. Atomic structure of the vimentin central alpha-helical domain and its implications for intermediate filament assembly. Proc. Natl Acad. Sci. USA. 109, 13620-13625 (2012).

38. Parry, D. A. Microdissection of the sequence and structure of intermediate filament chains. Adv. Protein Chem. 70, 113-142 (2005).

39. Meier, M. et al. Vimentin coil 1A-A molecular switch involved in the initiation of filament elongation. J. Mol. Biol. 390, 245-261 (2009).

40. Nicolet, S., Herrmann, H., Aebi, U. \& Strelkov, S. V. Atomic structure of vimentin coil 2. J. Struct. Biol. 170, 369-376 (2010).

41. Strelkov, S. V. \& Burkhard, P. Analysis of alpha-helical coiled coils with the program TWISTER reveals a structural mechanism for stutter compensation. J. Struct. Biol. 137, 54-64 (2002).

42. Schirmer, E. C. \& Gerace, L. The stability of the nuclear lamina polymer changes with the composition of lamin subtypes according to their individual binding strengths. J. Biol. Chem. 279, 42811-42817 (2004).

43. Kahraman, A. et al. Cross-link guided molecular modeling with ROSETTA. PLoS ONE 8, e73411 (2013).

44. Kahraman, A., Malmstrom, L. \& Aebersold, R. Xwalk: computing and visualizing distances in cross-linking experiments. Bioinformatics 27, 2163-2164 (2011).

45. Aziz, A., Hess, J. F., Budamagunta, M. S., FitzGerald, P. G. \& Voss, J. C. Head and rod 1 interactions in vimentin: identification of contact sites, structure, and changes with phosphorylation using site-directed spin labeling and electron paramagnetic resonance. J. Biol. Chem. 284, 7330-7338 (2009).

46. Gohara, R. et al. Phosphorylation of vimentin head domain inhibits interaction with the carboxyl-terminal end of alpha-helical rod domain studied by surface plasmon resonance measurements. FEBS Lett. 489, 182-186 (2001).

47. Matic, I. et al. Absolute SILAC-compatible expression strain allows Sumo-2 copy number determination in clinical samples. J. Proteome Res. 10, 4869-4875 (2011).

48. Makarov, A. A., Rizzotto, A., Meinke, P. \& Schirmer, E. C. Purification of lamins and soluble fragments of NETs. Methods Enzym. 569, 79-100 (2016).

49. Laue, T. M., Shah, B. D., Ridgeway, T. M. \& Pelletier, S. L. in Analytical Ultracentrifugation in Biochemistry and Polymer Science (eds Harding, S. E., Rowe, A. J. \& Horton J. C.) 90-125 (The Royal Society of Chemistry, London, 1992).

50. Schuck, P. Sedimentation analysis of noninteracting and self-associating solutes using numerical solutions to the Lamm equation. Biophys. J. 75, 1503-1512 (1998).

51. Brookes, E., Demeler, B. \& Rocco, M. Developments in the US-SOMO bead modeling suite: new features in the direct residue-to-bead method, improved grid routines, and influence of accessible surface area screening. Macromol. Biosci. 10, 746-753 (2010).

52. Rai, N. et al. SOMO (SOlution MOdeler) differences between X-Ray- and NMR-derived bead models suggest a role for side chain flexibility in protein hydrodynamics. Structure 13, 723-734 (2005).

53. Maiolica, A. et al. Structural analysis of multiprotein complexes by crosslinking, mass spectrometry, and database searching. Mol. Cell. Prot. 6, 2200-2211 (2007).

54. Ishihama, Y., Rappsilber, J. \& Mann, M. Modular stop and go extraction tips with stacked disks for parallel and multidimensional Peptide fractionation in proteomics. J. Proteome Res. 5, 988-994 (2006).

55. Rappsilber, J., Ishihama, Y. \& Mann, M. Stop and go extraction tips for matrix-assisted laser desorption/ionization, nanoelectrospray, and LC/MS sample pretreatment in proteomics. Anal. Chem. 75, 663-670 (2003).

56. Rappsilber, J., Mann, M. \& Ishihama, Y. Protocol for micro-purification, enrichment, pre-fractionation and storage of peptides for proteomics using StageTips. Nat. Protoc. 2, 1896-1906 (2007).

57. Chen, Z. A. et al. Architecture of the RNA polymerase II-TFIIF complex revealed by cross-linking and mass spectrometry. EMBO J. 29, 717-726 (2010).

58. Cox, J. \& Mann, M. MaxQuant enables high peptide identification rates, individualized p.p.b.-range mass accuracies and proteome-wide protein quantification. Nat. Biotechnol. 26, 1367-1372 (2008).

59. Fischer L. XiSearch (https://github.com/Rappsilber-Laboratory/XiSearch) (2017).

60. Lenz, S., Giese, S. H., Fischer, L. \& Rappsilber, J. In-search assignment of monoisotopic peaks improves the identification of cross-linked peptides. $J$. Proteome Res. 17, 3923-3931 (2018).

61. Fischer, L. \& Rappsilber, J. Quirks of error estimation in cross-linking/mass spectrometry. Anal. Chem. 89, 3829-3833 (2017).

62. Korfali, N., Florens, L. \& Schirmer, E. C. Isolation, proteomic analysis, and microscopy confirmation of the liver nuclear envelope proteome. Methods Mol. Biol. 1411, 3-44 (2016).
63. Dwyer, N. \& Blobel, G. A modified procedure for the isolation of a pore complex-lamina fraction from rat liver nuclei. J. Cell Biol. 70, 581-591 (1976).

64. Yang, L., Guan, T. \& Gerace, L. Integral membrane proteins of the nuclear envelope are dispersed throughout the endoplasmic reticulum during mitosis. J. Cell Biol. 137, 1199-1210 (1997).

65. Wisniewski, J. R., Zougman, A., Nagaraj, N. \& Mann, M. Universal sample preparation method for proteome analysis. Nat. Methods 6, 359-362 (2009).

66. Kessner, D., Chambers, M., Burke, R., Agus, D. \& Mallick, P. ProteoWizard: open source software for rapid proteomics tools development. Bioinformatics 24, 2534-2536 (2008).

67. Vizcaino, J. A. et al. 2016 update of the PRIDE database and its related tools. Nucleic Acids Res. 44, D447-D456 (2016).

68. MacLean, B. et al. Skyline: an open source document editor for creating and analyzing targeted proteomics experiments. Bioinformatics 26, (966-968 (2010).

69. Combe, C. W., Fischer, L. \& Rappsilber, J. xiNET: cross-link network maps with residue resolution. Mol. Cell. Prot. 14, 1137-1147 (2015).

70. Wood, C. W. et al. CCBuilder: an interactive web-based tool for building, designing and assessing coiled-coil protein assemblies. Bioinformatics 30, 3029-3035 (2014).

71. Arnold, K., Bordoli, L., Kopp, J. \& Schwede, T. The SWISS-MODEL workspace: a web-based environment for protein structure homology modelling. Bioinformatics 22, 195-201 (2006).

72. Bordoli, L. et al. Protein structure homology modeling using SWISS-MODEL workspace. Nat. Protoc. 4, 1-13 (2008).

73. Guex, N., Peitsch, M. C. \& Schwede, T. Automated comparative protein structure modeling with SWISS-MODEL and Swiss-PdbViewer: a historical perspective. Electrophoresis 30(Suppl 1), S162-S173 (2009).

74. Biasini, M. et al. SWISS-MODEL: modelling protein tertiary and quaternary structure using evolutionary information. Nucleic Acids Res. 42, W252-W258 (2014).

75. Zhang, Y. I-TASSER server for protein $3 \mathrm{D}$ structure prediction. BMC Bioinforma. 9, 40 (2008).

76. Yang, J. et al. The I-TASSER Suite: protein structure and function prediction. Nat. Methods 12, 7-8 (2015).

77. Roy, A., Kucukural, A. \& Zhang, Y. I-TASSER: a unified platform for automated protein structure and function prediction. Nat. Protoc. 5, 725-738 (2010).

78. Schrodinger, L. L. C. The PyMOL Molecular Graphics System. Version 1, 8 (2015).

79. Lyskov, S. \& Gray, J. J. The RosettaDock server for local protein-protein docking. Nucleic Acids Res. 36, W233-W238 (2008).

80. Lyskov, S. et al. Serverification of molecular modeling applications: the Rosetta Online Server that Includes Everyone (ROSIE). PLoS ONE 8, e63906 (2013).

81. Chaudhury, S. et al. Benchmarking and analysis of protein docking performance in Rosetta v3.2. PLoS ONE 6, e22477 (2011).

82. Herzog, F. et al. Structural probing of a protein phosphatase $2 \mathrm{~A}$ network by chemical cross-linking and mass spectrometry. Science 337, 1348-1352 (2012)

83. Gray, J. J. et al. Protein-protein docking with simultaneous optimization of rigid-body displacement and side-chain conformations. J. Mol. Biol. 331, 281-299 (2003).

84. Kumar, S. \& Nussinov, R. Close-range electrostatic interactions in proteins. Chembiochem 3, 604-617 (2002).

85. Dannenberg, J. J. An Introduction to Hydrogen Bonding By George A. Jeffrey (University of Pittsburgh) Oxford University Press: New York and Oxford, 1997. ix + 303 pp. \$60.00. ISBN 0-19-509549-9. J. Am. Chem. Soc. 120, 5604 (1998).

86. Webb, B. \& Sali, A. Comparative protein structure modeling using MODELLER. Curr. Protoc. Bioinforma. 54, 56 1-5 637 (2016).

87. Dolinsky, T. J., Nielsen, J. E., McCammon, J. A. \& Baker, N. A. PDB2PQR: an automated pipeline for the setup of Poisson-Boltzmann electrostatics calculations. Nucleic Acids Res. 32, W665-W667 (2004).

\section{Acknowledgements}

We thank Ueli Aebi for assistance setting up rotary shadowing, Zhuo Angel Chen for SILAC help, and Bill Earnshaw for critical input. This work was supported by Wellcome Senior Research Fellowships 095209 (E.C.S.) and 103139 (J.R.) and Wellcome Centre for Cell Biology core grant 092076. A.A.M. was supported by a University of Edinburgh Principal's PhD Scholarship.

\section{Author contributions}

Conceived/ designed experiments: A.A.M., E.C.S., J.R. Performed experiments: A.A.M., J.Z., D.R.H., C.S., A.S.S., C.C.P. Provided reagents/analysis tools: J.R., A.S.S. Wrote the manuscript: A.A.M., E.C.S. 


\section{Additional information}

Supplementary Information accompanies this paper at https://doi.org/10.1038/s41467019-11063-6.

Competing interests: The authors declare no competing interests.

Reprints and permission information is available online at http://npg.nature.com/ reprintsandpermissions/

Peer review information: Nature Communications thanks Florian Stengel and other anonymous reviewer(s) for their contribution to the peer review of this work. Peer reviewer reports are available.

Publisher's note: Springer Nature remains neutral with regard to jurisdictional claims in published maps and institutional affiliations. (c) (i) Open Access This article is licensed under a Creative Commons Attribution 4.0 International License, which permits use, sharing, adaptation, distribution and reproduction in any medium or format, as long as you give appropriate credit to the original author(s) and the source, provide a link to the Creative Commons license, and indicate if changes were made. The images or other third party material in this article are included in the article's Creative Commons license, unless indicated otherwise in a credit line to the material. If material is not included in the article's Creative Commons license and your intended use is not permitted by statutory regulation or exceeds the permitted use, you will need to obtain permission directly from the copyright holder. To view a copy of this license, visit http://creativecommons.org/ licenses/by/4.0/.

(C) The Author(s) 2019 